\title{
Hermite-Hadamard Type Inequalities for Interval-Valued Preinvex Functions via Fractional Integral Operators
}

\author{
Hari Mohan Srivastava ${ }^{1,2,3,4}$ (D). Soubhagya Kumar Sahoo ${ }^{5} \cdot$ Pshtiwan Othman Mohammed $^{6}$ (D) Dumitru Baleanu Dum $^{7,8}$ \\ Bibhakar Kodamasingh ${ }^{5}$
}

Received: 14 July 2021 / Accepted: 25 November 2021 / Published online: 19 January 2022

(c) The Author(s) 2022, corrected publication 2022

\begin{abstract}
In this article, the notion of interval-valued preinvex functions involving the Riemann-Liouville fractional integral is described. By applying this, some new refinements of the Hermite-Hadamard inequality for the fractional integral operator are presented. Some novel special cases of the presented results are discussed as well. Also, some examples are presented to validate our results. The established outcomes of our article may open another direction for different types of integral inequalities for fractional interval-valued functions, fuzzy interval-valued functions, and their associated optimization problems.
\end{abstract}

Keywords $\mathrm{H}-\mathrm{H}$ inequalities $\cdot$ Preinvex functions $\cdot$ Interval-valued functions $\cdot \mathrm{R}-\mathrm{L}$ fractional integral $\cdot$ Fractional integral inequalities

Mathematics Subject Classification $26 \mathrm{~A} 51 \cdot 26 \mathrm{~A} 33 \cdot 26 \mathrm{D} 07 \cdot 26 \mathrm{D} 10 \cdot 26 \mathrm{D} 15$

Pshtiwan Othman Mohammed pshtiwansangawi@gmail.com

$\triangle$ Dumitru Baleanu dumitru@cankaya.edu.tr

1 Department of Mathematics and Statistics, University of Victoria, Victoria, BC V8W 3R4, Canada

2 Department of Medical Research, China Medical University Hospital, China Medical University, Taizhong 40402, Taiwan, Republic of China

3 Department of Mathematics and Informatics, Azerbaijan University, 71 Jeyhun Hajibeyli Street, AZ1007 Baku, Azerbaijan

4 Section of Mathematics, International Telematic University Uninettuno, 00186 Rome, Italy

5 Department of Mathematics, Siksha O Anusandhan University, Bhubaneswar, Odisha 751030, India

6 Department of Mathematics, College of Education, University of Sulaimani, Kurdistan Region, Sulaymaniyah, Iraq

7 Department of Mathematics, Faculty of Arts and Sciences, Cankaya University, 06530 Ankara, Turkey

8 Institute of Space Sciences, P.O. Box, MG-23, Magurele-Bucharest, 76900 Măgurele, Romania

\section{Abbreviations}

$\mathrm{H}-\mathrm{H}$ Hermite-Hadamard

R-L Riemann-Liouville

\section{Introduction}

The idea of convexity has a lot of recognition in the theory of inequality, and assumes an essential part in probability theory, operations research, finance, decision-making, and numerical analysis. As of late, several generalizations related to convex functions have been set up. The idea of integral inequality is a fascinating area for research within mathematical analysis. Some fundamental integral inequalities are being utilized as a tool for fostering the subjective properties of convexity. Moreover, fractional calculus deals with derivatives and integrations of any fractional order. Recently, studies on integral inequalities using fractional operators have become an interesting topic for several mathematicians. Sarikaya et al. [1] started the trend of applying Riemann-Liouville (R-L) fractional integral operator for the classical convex function to refine the well-known Hermite-Hadamard $(\mathrm{H}-\mathrm{H})$ inequality. It is seen that fractional calculus aims at establishing mathematical models. 
A real valued function $\Upsilon: K \subseteq \Re \rightarrow \Re$ (set of real numbers) is said to be convex iff the following inequality satisfies:

$\Upsilon\left(z j_{1}+(1-z) j_{2}\right) \leq z \Upsilon\left(j_{1}\right)+(1-z) \Upsilon\left(j_{2}\right)$,

for all $\mathrm{j}_{1}, \mathrm{j}_{2} \in \mathrm{K}, \mathrm{z} \in[0,1]$.

Let $\Upsilon: \mathrm{K} \subseteq \mathfrak{R} \longrightarrow \Re$ be a convex function with $\mathrm{j}_{1}<\mathrm{j}_{2}$ and $\mathrm{j}_{1}, \mathrm{j}_{2} \in \mathrm{K}$. Then, the $\mathrm{H}-\mathrm{H}$ inequality is expressed as follows (see [2]):

$$
\begin{aligned}
\Upsilon\left(\frac{\mathrm{j}_{1}+\mathrm{j}_{2}}{2}\right) & \leqq \frac{1}{\mathrm{j}_{2}-\mathrm{j}_{1}} \int_{\mathrm{j}_{1}}^{\mathrm{j}_{2}} \Upsilon(x) d x \\
& \leqq \frac{\Upsilon\left(\mathrm{j}_{1}\right)+\Upsilon\left(\mathrm{j}_{2}\right)}{2} .
\end{aligned}
$$

In the recent past, the classical $\mathrm{H}-\mathrm{H}$ inequality (1.2) has been generalized and extended extensively by numerous mathematicians under the assumption of some interesting new definitions as a generalization of a convex function.

The concept of interval analysis was introduced in his book by the American mathematician Moore [3]. Soon after the publication of this book, many researchers started finding its applications in the field of robotics, computer graphics, scientific computations, signal processing, optimization, neural networking, and other fields. Over the recent past decades, several researchers incorporated this theory in the premises of different kinds of inequalities, for example (see [4-10]).

Definition 1.1 (see [11]) $\Upsilon: I^{\circ} \longrightarrow \mathcal{K}^{+}$is called an interval convex function if

$\Upsilon\left(z j_{1}+(1-z) j_{2}\right) \supseteq z \Upsilon\left(j_{1}\right)+(1-z) \Upsilon\left(j_{2}\right)$

holds for any $\mathrm{j}_{1}, \mathrm{j}_{2} \in I^{\circ}$ and $\mathrm{z} \in[0,1]$.

Very Recently, Zhao et al. [11] in the year 2018, for the first time, established the generalization of $\mathrm{H}-\mathrm{H}$ inequality in the frame of interval-valued analysis.

Let $\Upsilon: \mathrm{K} \subseteq \mathfrak{R} \longrightarrow \mathfrak{R}$ be an interval-valued convex function, such that $\Upsilon(x)=[\underline{\Upsilon}(x), \bar{\Upsilon}(x)]$ with $\Upsilon \in I R_{\left[\mathrm{j}_{1}, \mathrm{j}_{2}\right]}$; let $h:[0,1] \longrightarrow \Re$ be a non-negative function and $h\left(\frac{1}{2}\right) \neq 0$. If $\Upsilon$ is a $h$-convex function, then the $\mathrm{H}-\mathrm{H}$ inequality is expressed as follows (see [11]):

$$
\begin{aligned}
\frac{1}{2 h\left(\frac{1}{2}\right)} \Upsilon\left(\frac{\mathrm{j}_{1}+\mathrm{j}_{2}}{2}\right) & \supseteq \frac{1}{\mathrm{j}_{2}-\mathrm{j}_{1}}(I R) \int_{\mathrm{j}_{1}}^{\mathrm{j}_{2}} \Upsilon(x) d x \\
& \supseteq\left[\Upsilon\left(\mathrm{j}_{1}\right)+\Upsilon\left(\mathrm{j}_{2}\right)\right](I R) \int_{0}^{1} h(t) d t .
\end{aligned}
$$

In the last couple of years, several well-known inequalities like the Hermite-Hadamard inequality, the Ostrowski inequality, and the Minkowski inequality are presented with the help of interval analysis. Furthermore, recently, mathematicians have started establishing integral inequalities via interval-valued fractional operators. For example, Budak et al. [12] applied Riemann-Liouville fractional integral operator to study Hermite-Hadamard inequality and Pachpatte-type inequality as follows:

Theorem 1.1 Let $\Upsilon:\left[\mathrm{j}_{1}, \mathrm{j}_{2}\right] \longrightarrow \mathfrak{R}_{I_{\Gamma}}^{+}$be an interval-valued convex function, such that $\Upsilon(x)=[\underline{\Upsilon}(x), \bar{\Upsilon}(x)]$ and $\alpha>0$ Then, the $H-H$ inequality is expressed as follows:

$$
\begin{aligned}
\Upsilon\left(\frac{j_{1}+j_{2}}{2}\right) & \supseteq \frac{\Gamma(\alpha+1)}{2\left(j_{2}-j_{1}\right)^{\alpha}}\left[\widetilde{\mho}_{\left(j_{1}\right)^{+}}^{\alpha} \Upsilon\left(j_{2}\right)+\widetilde{J}_{\left(j_{2}\right)^{-}}^{\alpha} \Upsilon\left(j_{1}\right)\right] \\
& \supseteq \frac{\Upsilon\left(j_{1}\right)+\Upsilon\left(j_{2}\right)}{2} .
\end{aligned}
$$

Theorem 1.2 If $\Upsilon, \Theta:\left[\mathrm{j}_{1}, \mathrm{j}_{2}\right] \longrightarrow \mathfrak{R}_{I}^{+}$are two interval-valued convex functions, such that $\Upsilon(x)=[\underline{\Upsilon}(x), \bar{\Upsilon}(x)]$ and $\Theta(x)=[\underline{\Theta}(x), \bar{\Theta}(x)]$, then for $\alpha>0$ Then, the H-H inequality is expressed as follows:

$$
\begin{aligned}
& \frac{\Gamma(\alpha+1)}{2\left(\mathrm{j}_{2}-\mathrm{j}_{1}\right)^{\alpha}} \\
& {\left[\mathfrak{\mho}_{\left(\mathrm{j}_{1}\right)^{+}}^{\alpha} \Upsilon\left(\mathrm{j}_{2}\right) \Theta\left(\mathrm{j}_{2}\right)+\mathfrak{\Im}_{\left(\mathrm{j}_{2}\right)^{-}}^{\alpha} \Upsilon\left(\mathrm{j}_{1}\right) \Theta\left(\mathrm{j}_{1}\right)\right]} \\
& \supseteq\left[\frac{1}{2}-\frac{\alpha}{(\alpha+1)(\alpha+2)}\right] \\
& U\left(\mathrm{j}_{1}, \mathrm{j}_{2}\right)+\left[\frac{\alpha}{(\alpha+1)(\alpha+2)}\right] V\left(\mathrm{j}_{1}, \mathrm{j}_{2}\right),
\end{aligned}
$$

where

$U\left(\mathrm{j}_{1}, \mathrm{j}_{2}\right)=\left[\Upsilon\left(\mathrm{j}_{1}\right) \Theta\left(\mathrm{j}_{1}\right)+\Upsilon\left(\mathrm{j}_{2}\right) \Theta\left(\mathrm{j}_{2}\right)\right]$,

and

$V\left(\mathrm{j}_{1}, \mathrm{j}_{2}\right)=\left[\Upsilon\left(\mathrm{j}_{1}\right) \Theta\left(\mathrm{j}_{2}\right)+\Upsilon\left(\mathrm{j}_{2}\right) \Theta\left(\mathrm{j}_{1}\right)\right]$

Theorem 1.3 If $\Upsilon, \Theta:\left[\mathrm{j}_{1}, \mathrm{j}_{2}\right] \longrightarrow \mathfrak{R}_{I}^{+}$are two interval-valued convex functions, such that $\Upsilon(x)=[\underline{\Upsilon}(x), \bar{\Upsilon}(x)]$ and $\Theta(x)=[\underline{\Theta}(x), \bar{\Theta}(x)]$, then for $\alpha>0$ Then, the H-H inequality is expressed as follows:

$$
\begin{aligned}
& 2 \Upsilon\left(\frac{j_{1}+j_{2}}{2}\right) \Theta\left(\frac{j_{1}+j_{2}}{2}\right) \supseteq \frac{\Gamma(\alpha+1)}{2\left(j_{2}-j_{1}\right)^{\alpha}} \\
& {\left[\mathfrak{\Im}_{\left(\mathrm{j}_{1}\right)^{+}}^{\alpha} \Upsilon\left(\mathrm{j}_{2}\right) \Theta\left(\mathrm{j}_{2}\right)+\mathfrak{\Im}_{\left(\mathrm{j}_{2}\right)^{-}}^{\alpha} \Upsilon\left(\mathrm{j}_{1}\right) \Theta\left(\mathrm{j}_{1}\right)\right]} \\
& +\left[\frac{1}{2}-\frac{\alpha}{(\alpha+1)(\alpha+2)}\right] V\left(\mathrm{j}_{1}, \mathrm{j}_{2}\right) \\
& +\left[\frac{\alpha}{(\alpha+1)(\alpha+2)}\right] U\left(\mathrm{j}_{1}, \mathrm{j}_{2}\right) .
\end{aligned}
$$


Consequently, Liu et al. [13] improved the HermiteHadamard inequality and presented the following refinements via fractional operator:

Theorem 1.4 Let $\Upsilon \in \mathcal{I} \mathcal{L}_{\left[\mathrm{j}_{1}, \mathrm{j}_{2}\right]}$ and $\mathrm{j}_{1}, \mathrm{j}_{2} \in J^{\circ}$ with $0 \leq \mathrm{j}_{1}<\mathrm{j}_{2}$. If $\Upsilon$ be an interval-valued convex function and $\Psi\left(\mathrm{j}_{1}+\mathrm{j}_{2}\right)-x=\Psi(x) \geq 0$ holds, then

$$
\begin{aligned}
& \Upsilon\left(\frac{\mathrm{j}_{1}+\mathrm{j}_{2}}{2}\right)\left[\mathfrak{J}_{\left(\mathrm{j}_{1}\right)^{+}}^{\alpha} \Psi\left(\mathrm{j}_{2}\right)+\mathfrak{\Im}_{\left(\mathrm{j}_{2}\right)^{-}}^{\alpha} \Psi\left(\mathrm{j}_{1}\right)\right] \\
& \supseteq\left[\mathfrak{J}_{\left(\mathrm{j}_{1}\right)^{+}}^{\alpha}(\Upsilon \Psi)\left(\mathrm{j}_{2}\right)+\mathfrak{J}_{\left(\mathrm{j}_{2}\right)^{-}}^{\alpha}(\Upsilon \Psi)\left(\mathrm{j}_{1}\right)\right] \\
& \supseteq \frac{\Upsilon\left(\mathrm{j}_{1}\right)+\Upsilon\left(\mathrm{j}_{2}\right)}{2}\left[\mathfrak{J}_{\left(\mathrm{j}_{1}\right)^{+}}^{\alpha} \Psi\left(\mathrm{j}_{2}\right)+\mathfrak{J}_{\left(\mathrm{j}_{2}\right)^{-}}^{\alpha} \Psi\left(\mathrm{j}_{1}\right)\right] .
\end{aligned}
$$

In the same paper, the authors also introduced the concept of interval harmonically convex function, which reads as follows:

Definition 1.2 (see [13]) $\Upsilon: I^{\circ} \subseteq \Re \backslash 0 \longrightarrow \mathcal{K}^{+}$is called an interval harmonically convex function if

$\Upsilon\left(\frac{j_{1} j_{2}}{z j_{1}+(1-z) j_{2}}\right) \supseteq z \Upsilon\left(j_{2}\right)+(1-z) \Upsilon\left(j_{1}\right)$

holds for all $\mathrm{j}_{1}, \mathrm{j}_{2} \in I^{\circ}$ and $\mathrm{z} \in[0,1]$.

They also presented a generalization of the Hermite-Hadamard inequality for this new class of interval harmonically convex function as follows:

Theorem 1.5 Let $\Upsilon \in \mathcal{I L}_{\left[\mathrm{j}_{1}, \mathrm{j}_{2}\right]}$ and $\mathrm{j}_{1}, \mathrm{j}_{2} \in J^{\circ}$ with $0 \leq \mathrm{j}_{1}<\mathrm{j}_{2}$. If $\Upsilon$ be an interval harmonically convex function and $\Psi(x)=\frac{1}{x}, x \in\left[\frac{1}{j_{2}}, \frac{1}{j_{1}}\right]$, then

$$
\begin{aligned}
\Upsilon\left(\frac{2 \mathrm{j}_{1} \mathrm{j}_{2}}{\mathrm{j}_{1}+\mathrm{j}_{2}}\right) \supseteq & \frac{\Gamma(\alpha+1)\left(\mathrm{j}_{1} \mathrm{j}_{2}\right)^{\alpha}}{2\left(\mathrm{j}_{2}-\mathrm{j}_{1}\right)^{\alpha}} \\
& {\left[\mathfrak{\Im}_{\left(\frac{1}{\mathrm{j}_{1}}\right)^{-}}^{\alpha} \Upsilon \circ \Psi\left(\frac{1}{\mathrm{j}_{2}}\right)+\mathfrak{\Im}_{\left(\frac{1}{j_{2}}\right)^{+}}^{\alpha} \Upsilon \circ \Psi\left(\frac{1}{\mathrm{j}_{1}}\right)\right] } \\
& \supseteq \frac{\Upsilon\left(\mathrm{j}_{1}\right)+\Upsilon\left(\mathrm{j}_{2}\right)}{2} .
\end{aligned}
$$

As further refinements, An et al. [14] introduced interval $\left(h_{1}, h_{2}\right)$ - convex function, Nwaeze et al. [15] proved $\mathrm{H}-\mathrm{H}$ inequality for n-polynomial convex interval-valued function, Zhao et al. [16] introduced the notion of interval-valued coordinated convex function, and Ali et al. [17], Kalsoom et al. [18], and Tariboon et al. [19] refined this concept via quantum calculus. Recently, this concept is also generalized to convex fuzzy interval-valued functions by Khan et al. [20]. Interval-valued analysis has also been used in decision-making methods with fuzzy environment [21-23], multi-criterion decision-making with single-valued neutrosophic soft aggregation operator [24], multi-criterion decision-making with SVTrN Dombi aggregation function [25], and decisionmaking process based on GRA approach [26].

The objective of this paper is to study fractional integral inequalities for interval-valued preinvex functions. Specifically, we establish Hermite-Hadamard inequality of differintegrals $\left(\mathrm{j}_{1}+\frac{1}{2} \xi\left(\mathrm{j}_{2}, \mathrm{j}_{1}\right)\right)$ type via Riemann-Liouville fractional model. Furthermore, Pachpatte-type inequalities are also established for interval-valued preinvex functions using these fractional integrals. This paper is structured as follows: In Sect. 2, some basic notions about preinvexity and properties in the frame of interval-valued function are presented. Section 4 deals with presenting some novel $\mathrm{H}-\mathrm{H}$ - and Pachpatte-type inequalities for interval-valued preinvex function involving Riemann-Liouville fractional integral operator. Finally, in Sect. 5, conclusions and future scope of this paper are hinted at.

\section{Preliminaries}

In this section, first, we present some pre-requisite knowledge about the interval-valued functions, the theory of preinvexity, interval-valued integration, and interval-valued fractional integration, which is used extensively throughout the paper.

\subsection{Basic Properties of Interval-Valued Functions}

Here, in this subsection, we present some basic arithmetic about interval analysis, which will be very helpful throughout the paper

$[\mathrm{D}]=[\underline{\mathrm{D}}, \overline{\mathrm{D}}] \quad(x \in \mathfrak{R}, \underline{\mathrm{D}} \leqq x \leqq \overline{\mathrm{D}} ; x \in \mathfrak{R}) ;$

$\left[\mathrm{D}_{1}\right]+\left[\mathrm{D}_{2}\right]=\left[\underline{\mathrm{D}_{1}}, \overline{\mathrm{D}_{1}}\right]+\left[\underline{\mathrm{D}_{2}}, \overline{\mathrm{D}_{2}}\right]=\left[\underline{\mathrm{D}_{1}}+\underline{\mathrm{D}_{2}}, \overline{\mathrm{D}_{1}}+\overline{\mathrm{D}_{2}}\right] ;$

$\left[\mathrm{D}_{1}\right]-\left[\mathrm{D}_{2}\right]=\left[\underline{\mathrm{D}_{1}}, \overline{\mathrm{D}_{1}}\right]-\left[\underline{\mathrm{D}_{2}}, \overline{\mathrm{D}_{2}}\right]=\left[\underline{\mathrm{D}_{1}}-\underline{\mathrm{D}_{2}}, \overline{\mathrm{D}_{1}}-\overline{\mathrm{D}_{2}}\right]$;

$\mathrm{D}_{1} \cdot \mathrm{D}_{2}=[\min \mathrm{K}, \max \mathrm{K}]$,

where

$\min \mathrm{K}=\min \left\{\underline{\mathrm{D}_{1} \mathrm{D}_{2}}, \underline{\mathrm{D}_{1}} \overline{\mathrm{D}_{2}}, \overline{\mathrm{D}_{1}} \underline{\mathrm{D}_{2}}, \overline{\mathrm{D}_{1} \mathrm{D}_{2}}\right\}$

and

$\max K=\max \left\{\underline{D_{1} D_{2}}, \underline{D_{1}} \overline{D_{2}}, \overline{D_{1}} \underline{D_{2}}, \overline{D_{1} D_{2}}\right\}$.

Let $D_{1}$ be an interval, such that it does not contain 0 . Then

$\frac{1}{\mathrm{D}_{1}}=\left[\frac{1}{\overline{\mathrm{D}_{1}}}, \frac{1}{\mathrm{D}_{1}}\right]$

and 
$\frac{\mathrm{D}_{1}}{\mathrm{D}_{2}}=\mathrm{D}_{1} \cdot\left(\frac{1}{\mathrm{D}_{2}}\right)=\left\{\frac{\mathrm{d}_{1}}{\mathrm{~d}_{2}}: \mathrm{d}_{1} \in \mathrm{d}_{1} \quad\right.$ and $\left.\quad \mathrm{d}_{2} \in \mathrm{D}_{2}\right\}$.

\section{Scalar Multiplication}

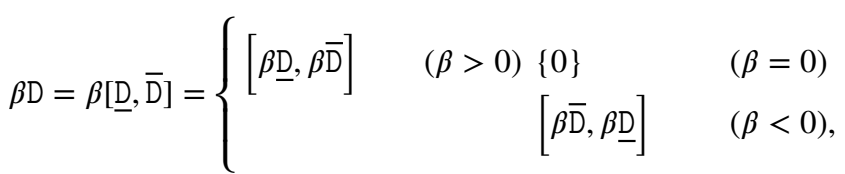

where $\beta \in \Re$.

Let $\Re_{I}, \Re_{I}^{+}$and $\Re_{I}^{-}$be the set of all closed intervals of $\Re$, the set of all positive closed intervals of $\mathfrak{R}$, and the set of all negative closed intervals of $\mathfrak{R}$, respectively. We now discuss some algebraic properties of interval arithmetic.

1. Associative Property for Addition: $\left(D_{1}+D_{2}\right)+D_{3}=D_{1}+\left(D_{2}+D_{3}\right)$ $\left(\forall D_{1}, D_{2}, D_{3} \in \Re_{I}\right)$.

2. Additive Element for Addition: $\mathrm{D}_{1}+0=0+\mathrm{D}_{1}=\mathrm{D}_{1}$ $\left(\forall D_{1} \in \mathfrak{R}_{I}\right)$.

3. Commutativity of Addition: $D_{1}+D_{2}=D_{2}+D_{1}$ $\left(\forall D_{1}, D_{2} \in \Re_{I}\right)$

4. Cancellation Law: $D_{1}+D_{3}=D_{2}+D_{3} \Longrightarrow D_{1}=D_{2}$ $\left(\forall D_{1}, D_{2}, D_{3} \in \Re_{I}\right)$.

5. Associativity of Multiplication: $\left(D_{1} \cdot D_{2}\right) \cdot D_{3}=D_{1} \cdot\left(D_{2} \cdot D_{3}\right)$ $\left(\forall D_{1}, D_{2}, D_{3} \in \Re_{I}\right)$

6. Commutativity of Multiplication : $D_{1} \cdot D_{2}=D_{2} \cdot D_{1}$ $\left(\forall D_{1}, D_{2} \in \Re_{I}\right)$.

7. Unit element : $\mathrm{D}_{1} \cdot 1=1 \cdot \mathrm{D}_{1}=\mathrm{D}_{1} \quad\left(\forall \mathrm{D}_{1} \in \mathfrak{R}_{I}\right)$.

8. Associate Law: $\gamma\left(\pi \mathrm{D}_{1}\right)=(\gamma \pi) \mathrm{D}_{1} \quad\left(\forall \mathrm{D}_{1} \in \mathfrak{R}_{I}\right.$ and $\left.\forall \gamma, \pi \in \mathfrak{R}\right)$.

9. First Distributive Law : $\gamma\left(\mathrm{D}_{1}+\mathrm{D}_{2}\right)=\gamma \mathrm{D}_{1}+\gamma \mathrm{D}_{2}$ $\left(\forall D_{1}, D_{2} \in \Re_{I}\right.$ and $\left.\forall \gamma \in \Re\right)$.

10. Second Distributive Law : $(\gamma+\pi) \mathrm{D}_{1}=\gamma \mathrm{D}_{1}+\pi \mathrm{D}_{1}$ $\left(\forall D_{1} \in \mathfrak{R}_{I}\right.$ and $\left.\forall \gamma, \pi \in \Re\right)$.

However, the distributive law does not always hold true.

\section{Example 2.1}

$\mathrm{D}_{1}=[1,2], \mathrm{D}_{2}=[2,4]$ and $\mathrm{D}_{3}=[-1,0]$.

$$
D_{1} \cdot\left(D_{2}+D_{3}\right)=[1,2] \cdot([2,4]+[-1,2])=[1,8],
$$

whereas

$D_{1} \cdot D_{2}+D_{1} \cdot D_{3}=[1,2] \cdot[2,4]+[1,2] \cdot[-1,0]=[0,8]$.

Recently, many researchers have shown their interest in the interval analysis and established some innovative inequalities in different directions (see, for details, [27-30]).

\subsection{The Concept of Preinvexity}

In the year 1981, Hanson [31] introduced the concept of invex functions with respect to bifunction $\xi(\cdot, \cdot)$ in mathematical programming. Soon after the publication of Hanson's work [31], Ben-Israel and Mond [32] explored the idea of invex sets and preinvex functions. The concept of preinvexity is more general than that of convexity.

In the year 1988, Weir and Mond [33] used the idea of invex sets to explore the concept of preinvexity.

Definition 2.1 (see [33]) Let $\mathrm{K} \neq \emptyset \in \mathfrak{R}$ be an invex set with respect to $\xi: \mathrm{K} \times \mathrm{K} \neq \emptyset \rightarrow \Re$. Then, the function $\Upsilon: \mathrm{K} \rightarrow \mathfrak{R}$ is said to be preinvex with respect to $\xi$ if

$\Upsilon\left(j_{2}+z \xi\left(j_{1}, j_{2}\right)\right) \leqq(z) \Upsilon\left(j_{1}\right)+(1-z) \Upsilon\left(j_{2}\right)$

$\left(\forall j_{1}, j_{2} \in \mathbb{X} ; z \in[0,1]\right)$.

The function $-\Upsilon$ is said to be preincave if and only if $\Upsilon$ is preinvex. We clearly see that if we choose

$\xi\left(\mathrm{j}_{1}, \mathrm{j}_{2}\right)=\mathrm{j}_{1}-\mathrm{j}_{2}$,

then, as a result, we attain the classical convex function from the more general form of preinvex function.

We also need the following hypothesis regarding the function $\xi$ which was studied by Mohan and Neogy [34].

Condition C: Let $\mathbb{X} \subset R^{n}$ be an open invex subset with respect to $\xi: \mathbb{X} \times \mathbb{X} \rightarrow \mathfrak{R}$. For any $\mathrm{j}_{1}, \mathrm{j}_{2} \in \mathbb{X}$ and $\mathrm{z} \in[0,1]$

$\xi\left(\mathrm{j}_{2}, \mathrm{j}_{2}+\mathrm{z} \xi\left(\mathrm{j}_{1}, \mathrm{j}_{2}\right)\right)=-\mathrm{z} \xi\left(\mathrm{j}_{1}, \mathrm{j}_{2}\right)$

and

$\xi\left(\mathrm{j}_{1}, \mathrm{j}_{2}+\mathrm{z} \xi\left(\mathrm{j}_{1}, \mathrm{j}_{2}\right)\right)=(1-\mathrm{z}) \xi\left(\mathrm{j}_{1}, \mathrm{j}_{2}\right)$.

In fact, for any $j_{1}, j_{2} \in \mathbb{X}$ and $z_{1}, z_{2} \in[0,1]$, we find from Condition $\mathrm{C}$ that

$\xi\left(j_{2}+z_{2} \xi\left(j_{1}, j_{2}\right), j_{2}+z_{1} \xi\left(j_{1}, j_{2}\right)\right)=\left(z_{2}-z_{1}\right) \xi\left(j_{1}, j_{2}\right)$.

In the year 2007, Noor [35] established and examined a new variant of $\mathrm{H}-\mathrm{H}$ type inequality for preinvex functions, which is stated below as Theorem 2.1 .

Theorem 2.1 Let $\Upsilon: \mathbb{X}=\left[\mathrm{j}_{1}, \mathrm{j}_{1}+\xi\left(\mathrm{j}_{2}, \mathrm{j}_{1}\right)\right] \rightarrow(0, \infty)$ be a preinvex function on the interval $\mathbb{X}^{\circ}$ of real numbers. Also, let $\mathrm{j}_{1}, \mathrm{j}_{2} \in \mathbb{X}$ with $\mathrm{j}_{1}<\mathrm{j}_{1}+\xi\left(\mathrm{j}_{1}, \mathrm{j}_{2}\right)$. Then, the following $H-H$ type inequalities for preinvex functions holds true :

$$
\begin{aligned}
\Upsilon\left(\frac{2 \mathrm{j}_{1}+\xi\left(\mathrm{j}_{2}, \mathrm{j}_{1}\right)}{2}\right) & \leqq \frac{1}{\xi\left(\mathrm{j}_{2}, \mathrm{j}_{1}\right)} \int_{\mathrm{j}_{1}}^{\mathrm{j}_{1}+\xi\left(\mathrm{j}_{2}, \mathrm{j}_{1}\right)} \Upsilon(x) d x \\
& \leqq \frac{\Upsilon\left(\mathrm{j}_{1}\right)+\Upsilon\left(\mathrm{j}_{2}\right)}{2} .
\end{aligned}
$$

For some detailed knowledge and perspectives about $\mathrm{H}-\mathrm{H}$ inequality in the frame of Preinvexity, we encourage interested readers to (see, for example, $[35,36]$ and the references cited therein). 
Motivated by the articles $[11,35,40]$ and above literature about interval analysis, preinvexity, and fractional calculus, we study some further refinements of $\mathrm{H}-\mathrm{H}$ - and Pachpattetype inequalities for interval-valued preinvex functions via $\mathrm{R}-\mathrm{L}$ fractional operators.

\subsection{Interval-Valued Integration}

Here, in this subsection, we present the Riemann integral operator for interval-valued functions.

Definition 2.2 Let $\Upsilon:\left[\mathrm{j}_{1}, \mathrm{j}_{2}\right]$ be an interval-valued function, such that $\Upsilon=[\Upsilon, \bar{\Upsilon}]$. Then, $\Upsilon$ is Riemann integrable on $\left[j_{1}, j_{2}\right]$ if and only if $\underline{\Upsilon}$ and $\bar{\Upsilon}$ are Riemann integrable on $\left[\mathrm{j}_{1}, \mathrm{j}_{2}\right]$, that is

$$
\text { (IR) } \begin{aligned}
& \int_{\mathrm{m}_{1}}^{\mathrm{m}_{2}} \Upsilon(a) d a \\
= & {\left[(R) \int_{\mathrm{m}_{1}}^{\mathrm{m}_{2}} \underline{\Upsilon}(a) d a,(R) \int_{\mathrm{m}_{1}}^{\mathrm{m}_{2}} \bar{\Upsilon}(a) d a\right] . }
\end{aligned}
$$

Definition 2.3 (see, for details, [37]; see also [1]) Let $\Upsilon \in \mathcal{L}\left[j_{1}, j_{2}\right]$. Then, the fractional integrals $\mathfrak{\Im}_{j_{1}^{+}}^{\alpha}$ and $\mathfrak{\Im}_{j_{2}^{-}}^{\alpha}$ of order $\alpha>0$ are defined as

$$
\widetilde{\mho}_{\mathrm{j}_{1}^{+}}^{\alpha} \Upsilon(x):=\frac{1}{\Gamma(\alpha)} \int_{\mathrm{j}_{1}}^{x}(x-\mathrm{z})^{\alpha-1} \Upsilon(\mathrm{z}) d \mathrm{z} \quad\left(0 \leqq \mathrm{j}_{1}<x<\mathrm{j}_{2}\right)
$$

and

$\mathfrak{\mho}_{\mathrm{j}_{2}^{-}}^{\alpha} \Upsilon(x):=\frac{1}{\Gamma(\alpha)} \int_{x}^{\mathrm{j}_{2}}(\mathrm{z}-x)^{\alpha-1} \Upsilon(\mathrm{z}) d \mathrm{z} \quad\left(0 \leqq \mathrm{j}_{2}<x<\mathrm{j}_{2}\right)$, respectively.

Corollary 2.1 Let $\Upsilon:\left[\mathrm{j}_{1}, \mathrm{j}_{2}\right]$ be an interval-valued function, such that $\Upsilon=[\underline{\Upsilon}, \bar{\Upsilon}]$ with $\underline{\Upsilon}, \bar{\Upsilon} \in \Re_{\left[\mathrm{j}_{1}, j_{2}\right]}$. Then

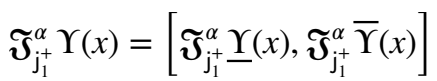

and

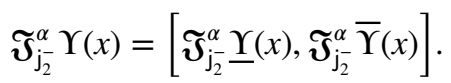

Proof The proof of Corollary 2.1 follows directly from Definition 2.2.

For other generalizations and detailed knowledge about fractional-calculus operators, the interested readers can see [37-40].

\section{H-H-Type Inequalities}

The principal objective and the main aim of this section are to establish a novel version of the $\mathrm{H}-\mathrm{H}$-type inequalities in the mode of interval-valued preinvex functions.

Theorem 3.1 Let $\mathrm{K} \subseteq \Re$ be an open invex subset with respect to $\xi: \mathrm{K} \times \mathrm{K} \rightarrow \mathfrak{R}$. Also, let $\mathrm{j}_{1}, \mathrm{j}_{2} \in \mathbb{X}$ with $\mathrm{j}_{2}+\xi\left(\mathrm{j}_{1}, \mathrm{j}_{2}\right) \leqq \mathrm{j}_{2}$. Suppose that $\Upsilon:\left[\mathrm{j}_{2}+\xi\left(\mathrm{j}_{1}, \mathrm{j}_{2}\right), \mathrm{j}_{2}\right] \longrightarrow \Re$ is an interval-valued preinvex function, such that $\Upsilon(\mathrm{z})=[\bar{\Upsilon}(\mathrm{z}), \underline{\Upsilon}(\mathrm{z})]$ and satisfies Condition $C$. Then, the following $H-H$ type inequalities hold true:

$$
\begin{aligned}
\Upsilon\left(j_{1}+\frac{1}{2} \xi\left(j_{2}, j_{1}\right)\right) \supseteq & \frac{2^{\alpha-1} \Gamma(\alpha+1)}{\xi^{\alpha}\left(j_{2}, j_{1}\right)} \\
& {\left[\mathfrak{\Im}_{\left(j_{1}+\frac{1}{2} \xi\left(j_{2}, j_{1}\right)\right)^{+}}^{\alpha} \Upsilon\left(j_{2}\right)+\mathfrak{J}_{\left(j_{1}+\frac{1}{2} \xi\left(j_{2}, j_{1}\right)\right)^{-}}^{\alpha} \Upsilon\left(j_{1}\right)\right] } \\
& \supseteq \frac{\Upsilon\left(j_{1}\right)+\Upsilon\left(j_{1}+\xi\left(j_{2}, j_{1}\right)\right)}{2} \supseteq \frac{\Upsilon\left(j_{1}\right)+\Upsilon\left(j_{2}\right)}{2} .
\end{aligned}
$$

Proof From the convexity theory, we have

$\Upsilon\left(x+\frac{1}{2} \xi(y, x)\right) \supseteq \frac{\Upsilon(x)+\Upsilon(y)}{2}$.

Choosing

$x=\mathrm{j}_{1}+\left(\frac{2-\mathrm{z}}{2}\right) \xi\left(\mathrm{j}_{2}, \mathrm{j}_{1}\right)$,

and

$y=\mathrm{j}_{1}+\frac{\mathrm{z}}{2} \xi\left(\mathrm{j}_{2}, \mathrm{j}_{1}\right)$.

It is seen that

$$
\begin{gathered}
\Upsilon\left(j_{1}+\left(\frac{2-z}{2}\right) \xi\left(j_{2}, j_{1}\right)+\frac{1}{2} \xi\left(j_{1}+\frac{z}{2} \xi\left(j_{2}, j_{1}\right), j_{1}+\left(\frac{2-z}{2}\right) \xi\left(j_{2}, j_{1}\right)\right)\right) \\
\supseteq \frac{\Upsilon\left(j_{1}+\left(\frac{2-z}{2}\right) \xi\left(j_{2}, j_{1}\right)\right)+\Upsilon\left(j_{1}+\frac{z}{2} \xi\left(j_{2}, j_{1}\right)\right)}{2} ;
\end{gathered}
$$

implies

$$
\begin{aligned}
& 2 \Upsilon\left(j_{1}+\frac{1}{2} \xi\left(j_{2}, j_{1}\right)\right) \supseteq \Upsilon\left(j_{1}+\left(\frac{2-z}{2}\right) \xi\left(j_{2}, j_{1}\right)\right) \\
& \quad+\Upsilon\left(j_{1}+\frac{z}{2} \xi\left(j_{2}, j_{1}\right)\right)
\end{aligned}
$$

Multiplying both sides of (3.2) by $z^{\alpha-1} \quad(\alpha>0)$ and integrating over the closed interval $[0,1]$, we obtain 


$$
\begin{aligned}
& 2(I R) \int_{0}^{1} \mathrm{z}^{\alpha-1} \Upsilon\left(\mathrm{j}_{1}+\frac{1}{2} \xi\left(\mathrm{j}_{2}, \mathrm{j}_{1}\right)\right) d \mathrm{z} \\
& \supseteq(I R) \int_{0}^{1} \mathrm{z}^{\alpha-1} \Upsilon\left(\mathrm{j}_{1}+\left(\frac{2-\mathrm{z}}{2}\right) \xi\left(\mathrm{j}_{2}, \mathrm{j}_{1}\right)\right) d \mathrm{z} \\
& \quad+(I R) \int_{0}^{1} \mathrm{z}^{\alpha-1} \Upsilon\left(\mathrm{j}_{1}+\frac{\mathrm{z}}{2} \xi\left(\mathrm{j}_{2}, \mathrm{j}_{1}\right)\right) d \mathrm{z} .
\end{aligned}
$$

Now, using Definition 2.2 and Definition 2.3, we get

$$
\begin{aligned}
& \Upsilon\left(\mathrm{j}_{1}+\frac{1}{2} \xi\left(\mathrm{j}_{2}, \mathrm{j}_{1}\right)\right) \supseteq \frac{2^{\alpha-1} \Gamma(\alpha+1)}{\xi^{\alpha}\left(\mathrm{j}_{2}, \mathrm{j}_{1}\right)} \\
& \quad\left[\mathfrak{J}_{\left(\mathrm{j}_{1}+\frac{1}{2} \xi\left(\mathrm{j}_{2}, \mathrm{j}_{1}\right)\right)^{+}}^{\alpha} \Upsilon\left(\mathrm{j}_{2}\right)+\mathfrak{\Im}_{\left(\mathrm{j}_{1}+\frac{1}{2} \xi\left(\mathrm{j}_{2}, \mathrm{j}_{1}\right)\right)^{-}}^{\alpha} \Upsilon\left(\mathrm{j}_{1}\right)\right] .
\end{aligned}
$$

This proves the first inequality asserted by Theorem 3.1.

For the proof of the second inequality in Theorem 3.1, we need the following results:

$$
\begin{aligned}
& 2(I R) \int_{0}^{1} \mathrm{z}^{\alpha-1} \Upsilon\left(\mathrm{j}_{1}+\frac{1}{2} \xi\left(\mathrm{j}_{2}, \mathrm{j}_{1}\right)\right) d \mathrm{z} \\
& =2\left[(R) \int_{0}^{1} \mathrm{z}^{\alpha-1} \bar{\Upsilon}\left(\mathrm{j}_{1}+\frac{1}{2} \xi\left(\mathrm{j}_{2}, \mathrm{j}_{1}\right)\right) d \mathrm{z},(R) \int_{0}^{1} \mathrm{z}^{\alpha-1} \underline{\Upsilon}\left(\mathrm{j}_{1}+\frac{1}{2} \xi\left(\mathrm{j}_{2}, \mathrm{j}_{1}\right)\right) d \mathrm{z}\right] \\
& =2\left[\bar{\Upsilon}\left(\mathrm{j}_{1}+\frac{1}{2} \xi\left(\mathrm{j}_{2}, \mathrm{j}_{1}\right)\right)(R) \int_{0}^{1} \mathrm{z}^{\alpha-1} d \mathrm{z}, \underline{\Upsilon}\left(\mathrm{j}_{1}+\frac{1}{2} \xi\left(\mathrm{j}_{2}, \mathrm{j}_{1}\right)\right)(R) \int_{0}^{1} \mathrm{z}^{\alpha-1} d \mathrm{z}\right] \\
& =\frac{2}{\alpha} \bar{\Upsilon}\left(\mathrm{j}_{1}+\frac{1}{2} \xi\left(\mathrm{j}_{2}, \mathrm{j}_{1}\right)\right), \frac{2}{\alpha} \underline{\Upsilon}\left(\mathrm{j}_{1}+\frac{1}{2} \xi\left(\mathrm{j}_{2}, \mathrm{j}_{1}\right)\right) \\
& =\frac{2}{\alpha} \Upsilon\left(j_{1}+\frac{1}{2} \xi\left(j_{2}, j_{1}\right)\right) \text {, }
\end{aligned}
$$

and

$$
\begin{aligned}
& \text { (IR) } \int_{0}^{1} \mathrm{z}^{\alpha-1} \Upsilon\left(\mathrm{j}_{1}+\left(\frac{2-\mathrm{z}}{2}\right) \xi\left(\mathrm{j}_{2}, \mathrm{j}_{1}\right)\right) d \mathrm{z} \\
& =(R) \int_{0}^{1} \mathrm{z}^{\alpha-1} \underline{\Upsilon}\left(\mathrm{j}_{1}+\left(\frac{2-\mathrm{z}}{2}\right) \xi\left(\mathrm{j}_{2}, \mathrm{j}_{1}\right)\right) d \mathrm{z},(R) \\
& \int_{0}^{1} \mathrm{z}^{\alpha-1} \bar{\Upsilon}\left(\mathrm{j}_{1}+\left(\frac{2-\mathrm{z}}{2}\right) \xi\left(\mathrm{j}_{2}, \mathrm{j}_{1}\right)\right) d z \\
& =(R) \int_{\mathrm{j}_{1}+\xi\left(\mathrm{j}_{2}, \mathrm{j}_{1}\right)}^{\left.\mathrm{j}_{1}+\frac{1}{2} \xi \mathrm{j}_{2}, \mathrm{j}_{1}\right)}\left(\frac{2}{\xi\left(\mathrm{j}_{2}, \mathrm{j}_{1}\right)}\left(\mathrm{j}_{2}-u\right)\right)^{\alpha-1} \underline{\Upsilon}(u) \frac{2 d u}{\xi\left(\mathrm{j}_{2}, \mathrm{j}_{1}\right)}, \\
& \text { (R) } \int_{\mathrm{j}_{1}+\xi\left(\mathrm{j}_{2}, \mathrm{j}_{1}\right)}^{\mathrm{j}_{1}+\frac{1}{2} \xi\left(\mathrm{j}_{2}, \mathrm{j}_{1}\right)}\left(\frac{2}{\xi\left(\mathrm{j}_{2}, \mathrm{j}_{1}\right)}\left(\mathrm{j}_{2}-u\right)\right)^{\alpha-1} \bar{\Upsilon}(u) \frac{2 d u}{\xi\left(\mathrm{j}_{2}, \mathrm{j}_{1}\right)}
\end{aligned}
$$

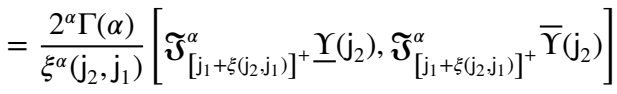

$$
\begin{aligned}
& =\frac{2^{\alpha} \Gamma(\alpha)}{\xi^{\alpha}\left(\mathrm{j}_{2}, \mathrm{j}_{1}\right)}\left[\mathfrak{\Im}_{\left[\mathrm{j}_{1}+\xi\left(\mathrm{j}_{2}, \mathrm{j}_{1}\right)\right]^{+}}^{\alpha} \Upsilon\left(\mathrm{j}_{2}\right)\right] \text {. }
\end{aligned}
$$

Similarly, we can show that

$$
\text { (IR) } \begin{aligned}
& \int_{0}^{1} \mathrm{z}^{\alpha-1} \Upsilon\left(\mathrm{j}_{1}+\frac{\mathrm{z}}{2} \xi\left(\mathrm{j}_{2}, \mathrm{j}_{1}\right)\right) d \mathrm{z} \\
= & \frac{2^{\alpha} \Gamma(\alpha)}{\xi^{\alpha}\left(\mathrm{j}_{2}, \mathrm{j}_{1}\right)}\left[\mathfrak{J}_{\left.\left[\mathrm{j}_{1}+\xi \mathrm{\xi}_{2}, \mathrm{j}_{1}\right)\right]^{+}}^{\alpha} \Upsilon\left(\mathrm{j}_{1}\right)\right] .
\end{aligned}
$$

Upon combining (3.3), (3.4), and (3.5), we have

$$
\Upsilon\left(j_{1}+\left(\frac{2-z}{2}\right) \xi\left(j_{2}, j_{1}\right)\right) \supseteq \frac{z}{2} \Upsilon\left(j_{1}\right)+\frac{2-z}{2} \Upsilon\left(j_{2}\right),
$$

and

$$
\begin{aligned}
& \Upsilon\left(\mathrm{j}_{1}+\left(\frac{\mathrm{z}}{2}\right) \xi\left(\mathrm{j}_{2}, \mathrm{j}_{1}\right)\right) \\
& \quad \supseteq \frac{2-\mathrm{z}}{2} \Upsilon\left(\mathrm{j}_{1}\right)+\frac{\mathrm{z}}{2} \Upsilon\left(\mathrm{j}_{2}\right) .
\end{aligned}
$$

Adding the last two inequalities, we have

$$
\begin{aligned}
& \Upsilon\left(\mathrm{j}_{1}+\left(\frac{2-\mathrm{z}}{2}\right) \xi\left(\mathrm{j}_{2}, \mathrm{j}_{1}\right)\right) \\
& \quad+\Upsilon\left(\mathrm{j}_{1}+\left(\frac{\mathrm{z}}{2}\right) \xi\left(\mathrm{j}_{2}, \mathrm{j}_{1}\right)\right) \\
& \quad \supseteq \Upsilon\left(\mathrm{j}_{1}\right)+\Upsilon\left(\mathrm{j}_{1}+\xi\left(\mathrm{j}_{2}, \mathrm{j}_{1}\right)\right) .
\end{aligned}
$$

Multiplying both sides of the above inequality by $z^{\alpha-1}(\alpha>0)$ and integrating over the closed interval $[0,1]$, we find that

$$
\begin{aligned}
& \text { (IR) } \int_{0}^{1} \mathrm{z}^{\alpha-1} \Upsilon\left(\mathrm{j}_{1}+\left(\frac{2-\mathrm{z}}{2}\right) \xi\left(\mathrm{j}_{2}, \mathrm{j}_{1}\right)\right) d \mathrm{z} \\
& +(I R) \int_{0}^{1} \mathrm{z}^{\alpha-1} \Upsilon\left(\mathrm{j}_{1}+\left(\frac{\mathrm{z}}{2}\right) \xi\left(\mathrm{j}_{2}, \mathrm{j}_{1}\right)\right) d \mathrm{z} \\
& \supseteq(I R) \int_{0}^{1} \mathrm{z}^{\alpha-1}\left[\Upsilon\left(\mathrm{j}_{1}\right)+\Upsilon\left(\mathrm{j}_{1}+\xi\left(\mathrm{j}_{2}, \mathrm{j}_{1}\right)\right)\right] d \mathrm{z} .
\end{aligned}
$$

And 


$$
\begin{aligned}
& \text { (IR) } \int_{0}^{1} \mathrm{z}^{\alpha-1}\left[\Upsilon\left(\mathrm{j}_{1}\right)+\Upsilon\left(\mathrm{j}_{1}+\xi\left(\mathrm{j}_{2}, \mathrm{j}_{1}\right)\right)\right] d \mathrm{z} \\
&=(R) \int_{0}^{1} \mathrm{z}^{\alpha-1}\left[\underline{\Upsilon}\left(\mathrm{j}_{1}\right)+\underline{\Upsilon}\left(\mathrm{j}_{1}+\xi\left(\mathrm{j}_{2}, \mathrm{j}_{1}\right)\right)\right] d z, \\
&(R) \int_{0}^{1} \mathrm{z}^{\alpha-1}\left[\bar{\Upsilon}\left(\mathrm{j}_{1}\right)+\bar{\Upsilon}\left(\mathrm{j}_{1}+\xi\left(\mathrm{j}_{2}, \mathrm{j}_{1}\right)\right)\right] d \mathrm{z} \\
&= \frac{1}{\alpha}\left[\underline{\Upsilon}\left(\mathrm{j}_{1}\right)+\underline{\Upsilon}\left(\mathrm{j}_{1}+\xi\left(\mathrm{j}_{2}, \mathrm{j}_{1}\right)\right)\right], \frac{1}{\alpha}\left[\bar{\Upsilon}\left(\mathrm{j}_{1}\right)+\bar{\Upsilon}\left(\mathrm{j}_{1}+\xi\left(\mathrm{j}_{2}, \mathrm{j}_{1}\right)\right)\right] \\
&= \frac{1}{\alpha}\left[\Upsilon\left(\mathrm{j}_{1}\right)+\Upsilon\left(\mathrm{j}_{1}+\xi\left(\mathrm{j}_{2}, \mathrm{j}_{1}\right)\right)\right] .
\end{aligned}
$$

If we substitute from (3.4), (3.5), and (3.8) into (3.7), we have

$$
\begin{aligned}
& \frac{2^{\alpha-1} \Gamma(\alpha+1)}{\xi^{\alpha}\left(\mathrm{j}_{2}, \mathrm{j}_{1}\right)} \\
& {\left[\mathfrak{\Im}_{\left(\mathrm{j}_{1}+\frac{1}{2} \xi\left(\mathrm{j}_{2}, \mathrm{j}_{1}\right)\right)^{+}}^{\alpha} \Upsilon\left(\mathrm{j}_{2}\right)+\mathfrak{\Im}_{\left(\mathrm{j}_{1}+\frac{1}{2} \xi\left(\mathrm{j}_{2}, \mathrm{j}_{1}\right)\right.}^{\alpha}\right)} \\
& \left.\quad \supseteq \frac{\Upsilon\left(\mathrm{j}_{1}\right)+\Upsilon\left(\mathrm{j}_{1}\right)}{2}+\xi\left(\mathrm{j}_{2}, \mathrm{j}_{1}\right)\right) \\
& 2
\end{aligned}
$$

This completes the rest of the proof of Theorem 3.1.

Remark 3.1 When we choose $\xi\left(\mathrm{j}_{2}, \mathrm{j}_{1}\right)=\mathrm{j}_{2}-\mathrm{j}_{1}$, Theorem 3.1 yields

$$
\begin{aligned}
& \Upsilon\left(\frac{j_{1}+j_{2}}{2}\right) \supseteq \frac{2^{\alpha-1} \Gamma(\alpha+1)}{\left(j_{2}-j_{1}\right)^{\alpha}} \\
& {\left[\mathfrak{\Im}_{\left(\frac{j_{1}+j_{2}}{2}\right)^{+}} \Upsilon\left(j_{2}\right)+\mathfrak{J}_{\left(\frac{j_{1}+j_{2}}{2}\right)^{\alpha}}^{\alpha} \Upsilon\left(j_{1}\right)\right]} \\
& \supseteq \frac{\Upsilon\left(j_{1}\right)+\Upsilon\left(j_{2}\right)}{2} .
\end{aligned}
$$

Remark 3.2 If we choose $\alpha=1$ in the assertion (3.1) of Theorem 3.1, we have

$$
\begin{aligned}
& \Upsilon\left(\frac{2 \mathrm{j}_{1}+\xi\left(\mathrm{j}_{2}, \mathrm{j}_{1}\right)}{2}\right) \supseteq \frac{1}{\xi\left(\mathrm{j}_{2}, \mathrm{j}_{1}\right)} \int_{\mathrm{j}_{1}}^{\mathrm{j}_{1}+\xi\left(\mathrm{j}_{2}, \mathrm{j}_{1}\right)} \Upsilon(x) d x \\
& \quad \supseteq \frac{\Upsilon\left(\mathrm{j}_{1}\right)+\Upsilon\left(\mathrm{j}_{2}\right)}{2} .
\end{aligned}
$$

Theorem 3.2 Let $\mathrm{K} \subseteq \Re$ be an open invex subset with respect to $\xi: \mathrm{K} \times \mathrm{K} \rightarrow \Re$. Also, let $\mathrm{j}_{1}, \mathrm{j}_{2} \in \mathbb{X}$ with $\mathrm{j}_{2}+\xi\left(\mathrm{j}_{1}, \mathrm{j}_{2}\right) \leqq \mathrm{j}_{2}$. Suppose that $\Upsilon, \Theta:\left[\mathrm{j}_{2}+\xi\left(\mathrm{j}_{1}, \mathrm{j}_{2}\right), \mathrm{j}_{2}\right] \longrightarrow \Re$ $i s$ an interval-valued preinvex function, such that $\Upsilon(\mathrm{z})=[\bar{\Upsilon}(\mathrm{z}), \underline{\Upsilon}(\mathrm{z})]$ and $\Theta(\mathrm{z})=[\bar{\Theta}(\mathrm{z}), \underline{\Theta}(\mathrm{z})]$. If $\Upsilon, \Theta \in \mathcal{L}\left[\mathrm{j}_{1}, \mathrm{j}_{2}\right]$ and if $\xi$ satisfies Condition $C$, then the following $\mathrm{H}-\mathrm{H}$ type inequalities hold true:

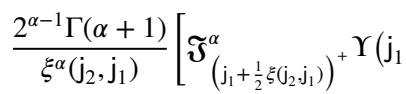

$\left.\left.+\xi\left(\mathrm{j}_{2}, \mathrm{j}_{1}\right)\right) \cdot \Theta\left(\mathrm{j}_{1}+\xi\left(\mathrm{j}_{2}, \mathrm{j}_{1}\right)\right)+\mathfrak{\Im}_{\left(\mathrm{j}_{1}+\frac{1}{2} \xi\left(\mathrm{j}_{2}, \mathrm{j}_{1}\right)\right.}^{\alpha}-\mathrm{Y}^{\left.\mathrm{j}_{1}\right)} \cdot \Theta\left(\mathrm{j}_{1}\right)\right]$

$\supseteq \frac{\alpha}{4}\left[\frac{2}{\alpha}-\frac{2}{\alpha+1}+\frac{1}{\alpha+2}\right] U\left(\mathrm{j}_{1}, \mathrm{j}_{2}\right)+\frac{\alpha}{4}\left[\frac{\alpha-3}{(\alpha+1)(\alpha+2)}\right] V\left(\mathrm{j}_{1}, \mathrm{j}_{2}\right)$,

where

$U\left(\mathrm{j}_{1}, \mathrm{j}_{2}\right)=\left[\Upsilon\left(\mathrm{j}_{1}\right) \Theta\left(\mathrm{j}_{1}\right)+\Upsilon\left(\mathrm{j}_{1}+\xi\left(\mathrm{j}_{2}, \mathrm{j}_{1}\right)\right) \Theta\left(\mathrm{j}_{1}+\xi\left(\mathrm{j}_{2}, \mathrm{j}_{1}\right)\right)\right]$

and

$V\left(\mathrm{j}_{1}, \mathrm{j}_{2}\right)=\left[\Upsilon\left(\mathrm{j}_{1}\right) \Theta\left(\mathrm{j}_{1}+\xi\left(\mathrm{j}_{2}, \mathrm{j}_{1}\right)\right)+\Upsilon\left(\mathrm{j}_{1}+\xi\left(\mathrm{j}_{2}, \mathrm{j}_{1}\right)\right) \Theta\left(\mathrm{j}_{1}\right)\right]$.

Proof Since $\Upsilon$ and $\Theta$ are interval-valued preinvex functions, we have

$$
\begin{aligned}
& \Upsilon\left(\mathrm{j}_{1}+\left(\frac{2-\mathrm{z}}{2}\right) \xi\left(\mathrm{j}_{2}, \mathrm{j}_{1}\right)\right) \\
& \quad \supseteq \frac{\mathrm{z}}{2} \Upsilon\left(\mathrm{j}_{1}\right)+\frac{2-\mathrm{z}}{2} \Upsilon\left(\mathrm{j}_{2}\right)
\end{aligned}
$$

and

$$
\begin{array}{r}
\Theta\left(\mathrm{j}_{1}+\left(\frac{2-\mathrm{z}}{2}\right) \xi\left(\mathrm{j}_{2}, \mathrm{j}_{1}\right)\right) \\
\supseteq \frac{\mathrm{z}}{2} \Theta\left(\mathrm{j}_{1}\right)+\frac{2-\mathrm{z}}{2} \Theta\left(\mathrm{j}_{2}\right) .
\end{array}
$$

Multiplying both the above inequalities, it is readily seen that

$$
\begin{gathered}
\Upsilon\left(\mathrm{j}_{1}+\left(\frac{2-\mathrm{z}}{2}\right) \xi\left(\mathrm{j}_{2}, \mathrm{j}_{1}\right)\right) \cdot \Theta\left(\mathrm{j}_{1}+\left(\frac{2-\mathrm{z}}{2}\right) \xi\left(\mathrm{j}_{2}, \mathrm{j}_{1}\right)\right) \\
\supseteq\left(\frac{\mathrm{z}}{2}\right)^{2} \Upsilon\left(\mathrm{j}_{1}\right) \Theta\left(\mathrm{j}_{1}\right)+\left(\frac{2-\mathrm{z}}{2}\right)^{2} \\
\Upsilon\left(\mathrm{j}_{1}+\xi\left(\mathrm{j}_{2}, \mathrm{j}_{1}\right)\right) \Theta\left(\mathrm{j}_{1}+\xi\left(\mathrm{j}_{2}, \mathrm{j}_{1}\right)\right) \\
\quad+\left(\frac{\mathrm{z}}{2}\right)\left(\frac{2-\mathrm{z}}{2}\right)\left[\Upsilon\left(\mathrm{j}_{1}\right) \Theta\left(\mathrm{j}_{1}+\xi\left(\mathrm{j}_{2}, \mathrm{j}_{1}\right)\right)\right. \\
\left.\quad \Upsilon\left(\mathrm{j}_{1}+\xi\left(\mathrm{j}_{2}, \mathrm{j}_{1}\right)\right) \Theta\left(\mathrm{j}_{1}\right)\right] .
\end{gathered}
$$

Similarly, we can observe that

$\Upsilon\left(\mathrm{j}_{1}+\left(\frac{\mathrm{z}}{2}\right) \xi\left(\mathrm{j}_{2}, \mathrm{j}_{1}\right)\right) \supseteq \frac{2-\mathrm{z}}{2} \Upsilon\left(\mathrm{j}_{1}\right)+\frac{\mathrm{z}}{2} \Upsilon\left(\mathrm{j}_{2}\right)$

and

$\Theta\left(\mathrm{j}_{1}+\left(\frac{\mathrm{z}}{2}\right) \xi\left(\mathrm{j}_{2}, \mathrm{j}_{1}\right)\right) \supseteq \frac{2-\mathrm{z}}{2} \Theta\left(\mathrm{j}_{1}\right)+\frac{\mathrm{z}}{2} \Theta\left(\mathrm{j}_{2}\right)$.

Consequently, we also have 
$\Upsilon\left(\mathrm{j}_{1}+\left(\frac{\mathrm{z}}{2}\right) \xi\left(\mathrm{j}_{2}, \mathrm{j}_{1}\right)\right) \cdot \Theta\left(\mathrm{j}_{1}+\left(\frac{\mathrm{z}}{2}\right) \xi\left(\mathrm{j}_{2}, \mathrm{j}_{1}\right)\right)$

$\supseteq\left(\frac{2-z}{2}\right)^{2} \Upsilon\left(j_{1}\right) \Theta\left(j_{1}\right)+\left(\frac{z}{2}\right)^{2}$

$\Upsilon\left(\mathrm{j}_{1}+\xi\left(\mathrm{j}_{2}, \mathrm{j}_{1}\right)\right) \Theta\left(\mathrm{j}_{1}+\xi\left(\mathrm{j}_{2}, \mathrm{j}_{1}\right)\right)+\left(\frac{\mathrm{z}}{2}\right)\left(\frac{2-\mathrm{z}}{2}\right)$

$\left[\Upsilon\left(j_{1}\right) \Theta\left(j_{1}+\xi\left(j_{2}, j_{1}\right)\right)+\Upsilon\left(j_{1}+\xi\left(j_{2}, j_{1}\right)\right) \Theta\left(j_{1}\right)\right]$.

Upon adding Eqs. (3.10) and (3.11), we get

$$
\begin{aligned}
& \Upsilon\left(j_{1}+\left(\frac{2-z}{2}\right) \xi\left(j_{2}, j_{1}\right)\right) \cdot \Theta\left(j_{1}+\left(\frac{2-z}{2}\right) \xi\left(j_{2}, j_{1}\right)\right) \\
& +\Upsilon\left(j_{1}+\left(\frac{z}{2}\right) \xi\left(j_{2}, j_{1}\right)\right) \cdot \Theta\left(j_{1}+\left(\frac{z}{2}\right) \xi\left(j_{2}, j_{1}\right)\right) \\
& \supseteq\left(\frac{z}{2}\right)^{2}\left[\Upsilon\left(j_{1}\right) \Theta\left(j_{1}\right)+\Upsilon\left(j_{1}+\xi\left(j_{2}, j_{1}\right)\right) \Theta\left(j_{1}+\xi\left(j_{2}, j_{1}\right)\right)\right] \\
& +\left(\frac{2-z}{2}\right)^{2}\left[\Upsilon\left(j_{1}\right) \Theta\left(j_{1}\right)+\Upsilon\left(j_{1}+\xi\left(j_{2}, j_{1}\right)\right) \Theta\left(j_{1}+\xi\left(j_{2}, j_{1}\right)\right)\right] \\
& +2\left(\frac{z}{2}\right)\left(\frac{2-z}{2}\right)\left[\Upsilon\left(j_{1}\right) \Theta\left(j_{1}+\xi\left(j_{2}, j_{1}\right)\right)+\Upsilon\left(j_{1}+\xi\left(j_{2}, j_{1}\right)\right) \Theta\left(j_{1}\right)\right] \\
& =U\left(j_{1}, j_{2}\right)\left[\left(\frac{z}{2}\right)^{2}+\left(\frac{2-z}{2}\right)^{2}\right]+2 V\left(j_{1}, j_{2}\right)\left(\frac{z}{2}\right)\left(\frac{2-z}{2}\right) .
\end{aligned}
$$

Now, if we multiply both sides of the inequality (3.12) by $z^{\alpha-1}(\alpha>0)$ and integrating over the closed interval $[0,1]$, we find that

(IR) $\int_{0}^{1} \mathrm{z}^{\alpha-1} \Upsilon\left(\mathrm{j}_{1}+\left(\frac{2-\mathrm{z}}{2}\right) \xi\left(\mathrm{j}_{2}, \mathrm{j}_{1}\right)\right) \cdot \Theta\left(\mathrm{j}_{1}+\left(\frac{2-\mathrm{z}}{2}\right) \xi\left(\mathrm{j}_{2}, \mathrm{j}_{1}\right)\right) d \mathrm{z}$ $+(I R) \int_{0}^{1} \mathrm{z}^{\alpha-1} \Upsilon\left(\mathrm{j}_{1}+\left(\frac{\mathrm{z}}{2}\right) \xi\left(\mathrm{j}_{2}, \mathrm{j}_{1}\right)\right) \cdot \Theta\left(\mathrm{j}_{1}+\left(\frac{\mathrm{z}}{2}\right) \xi\left(\mathrm{j}_{2}, \mathrm{j}_{1}\right)\right) d \mathrm{z}$

$\supseteq U\left(\mathrm{j}_{1}, \mathrm{j}_{2}\right)(I R) \int_{0}^{1} \mathrm{z}^{\alpha-1}\left[\left(\frac{\mathrm{z}}{2}\right)^{2}+\left(\frac{2-\mathrm{z}}{2}\right)^{2}\right] d \mathrm{z}$

$+2 V\left(\mathrm{j}_{1}, \mathrm{j}_{2}\right)(I R) \int_{0}^{1} \mathrm{z}^{\alpha-1}\left(\frac{\mathrm{z}}{2}\right)\left(\frac{2-\mathrm{z}}{2}\right) d \mathrm{z}$.

Using Definition 2.2 and Definition 2.3, we thus obtain

(IR) $\int_{0}^{1} \mathrm{z}^{\alpha-1} \Upsilon\left(\mathrm{j}_{1}+\left(\frac{2-\mathrm{z}}{2}\right) \xi\left(\mathrm{j}_{2}, \mathrm{j}_{1}\right)\right) \cdot \Theta\left(\mathrm{j}_{1}+\left(\frac{2-\mathrm{z}}{2}\right) \xi\left(\mathrm{j}_{2}, \mathrm{j}_{1}\right)\right) d \mathrm{z}$ $=\frac{2^{\alpha} \Gamma(\alpha)}{\xi^{\alpha}\left(\mathrm{j}_{2}, \mathrm{j}_{1}\right)}\left[\mathfrak{\Im}_{\left.\left(\mathrm{j}_{1}+\frac{1}{2} \xi \mathrm{j}_{2}, \mathrm{j}_{1}\right)\right)^{+}}^{\alpha} \Upsilon\left(\mathrm{j}_{1}+\xi\left(\mathrm{j}_{2}, \mathrm{j}_{1}\right)\right) \cdot \Theta\left(\mathrm{j}_{1}+\xi\left(\mathrm{j}_{2}, \mathrm{j}_{1}\right)\right)\right]$.

Similarly, we can show that

$$
\begin{gathered}
\text { (IR) } \int_{0}^{1} \mathrm{z}^{\alpha-1} \Upsilon\left(\mathrm{j}_{1}+\left(\frac{\mathrm{z}}{2}\right) \xi\left(\mathrm{j}_{2}, \mathrm{j}_{1}\right)\right) \cdot \Theta\left(\mathrm{j}_{1}+\left(\frac{\mathrm{z}}{2}\right) \xi\left(\mathrm{j}_{2}, \mathrm{j}_{1}\right)\right) d \mathrm{z} \\
=\frac{2^{\alpha} \Gamma(\alpha)}{\xi^{\alpha}\left(\mathrm{j}_{2}, \mathrm{j}_{1}\right)}\left[\widetilde{\mho}_{\left(\mathrm{j}_{1}+\frac{1}{2} \xi\left(\mathrm{j}_{2}, \mathrm{j}_{1}\right)\right)^{-}}^{\alpha} \Upsilon\left(\mathrm{j}_{1}\right) \cdot \Theta\left(\mathrm{j}_{1}\right)\right]
\end{gathered}
$$

$$
\text { (IR) } \begin{aligned}
& \int_{0}^{1} \mathrm{z}^{\alpha-1}\left[\left(\frac{\mathrm{z}}{2}\right)^{2}+\left(\frac{2-\mathrm{z}}{2}\right)^{2}\right] d \mathrm{z} \\
= & \frac{1}{2}\left[\frac{2}{\alpha}-\frac{2}{\alpha+1}+\frac{1}{\alpha+2}\right] .
\end{aligned}
$$

And

(IR) $\int_{0}^{1} z^{\alpha-1}\left(\frac{z}{2}\right)\left(\frac{2-z}{2}\right) d z=\frac{1}{4}\left[\frac{\alpha-3}{(\alpha+1)(\alpha+2)}\right]$.

Finally, by making use of Eqs. (3.13), (3.14), (3.15), and (3.16) in (3.12), we are led to the desired result as asserted by Theorem 3.2 .

Remark 3.3 When we choose $\xi\left(\mathrm{j}_{2}, \mathrm{j}_{1}\right)=\mathrm{j}_{2}-\mathrm{j}_{1}$, Theorem 3.2 yields

$$
\begin{array}{r}
\frac{2^{\alpha-1} \Gamma(\alpha+1)}{\left(\mathrm{j}_{2}-\mathrm{j}_{1}\right)^{\alpha}}\left[\mathfrak{\Im}_{\left(\frac{\mathrm{j}_{1}+\mathrm{j}_{2}}{2}\right)^{+}}^{\alpha} \Upsilon\left(\mathrm{j}_{2}\right) \Theta\left(\mathrm{j}_{2}\right)+\mathfrak{\Im}_{\left(\frac{\mathrm{j}_{1}+\mathrm{j}_{2}}{2}\right)^{-}}^{\alpha} \Upsilon\left(\mathrm{j}_{1}\right) \Theta\left(\mathrm{j}_{1}\right)\right. \\
\supseteq \frac{\alpha}{4}\left[\frac{2}{\alpha}-\frac{2}{\alpha+1}+\frac{1}{\alpha+2}\right] U\left(\mathrm{j}_{1}, \mathrm{j}_{2}\right)+\frac{\alpha}{4}\left[\frac{\alpha-3}{(\alpha+1)(\alpha+2)}\right] V\left(\mathrm{j}_{1}, \mathrm{j}_{2}\right) .
\end{array}
$$

Theorem 3.3 Let $\mathrm{K} \subseteq \Re$ be an open invex subset with respect to $\xi: \mathrm{K} \times \mathrm{K} \rightarrow \mathfrak{R}$. Also, let $\mathrm{j}_{1}, \mathrm{j}_{2} \in \mathbb{X}$ with

$\mathrm{j}_{2}+\xi\left(\mathrm{j}_{1}, \mathrm{j}_{2}\right) \leqq \mathrm{j}_{2}$.

Suppose that $\Upsilon, \Theta:\left[\mathrm{j}_{2}+\xi\left(\mathrm{j}_{1}, \mathrm{j}_{2}\right), \mathrm{j}_{2}\right] \longrightarrow \mathfrak{R}$ is an intervalvalued preinvex function, such that

$\Upsilon(z)=[\bar{\Upsilon}(z), \underline{\Upsilon}(z)] \quad$ and $\quad \Theta(z)=[\bar{\Theta}(z), \underline{\Theta}(z)]$.

If $\Upsilon, \Theta \in \mathcal{L}\left[\mathrm{j}_{1}, \mathrm{j}_{2}\right]$ and if $\xi$ satisfies Condition $C$, then the following $\mathrm{H}-\mathrm{H}$ type inequalities hold true:

$$
\begin{gathered}
\Upsilon\left(\mathrm{j}_{1}+\frac{1}{2} \xi\left(\mathrm{j}_{2}, \mathrm{j}_{1}\right)\right) \cdot \Theta\left(\mathrm{j}_{1}+\frac{1}{2} \xi\left(\mathrm{j}_{2}, \mathrm{j}_{1}\right)\right) \\
\supseteq \frac{2^{\alpha-1} \Gamma(\alpha+1)}{\xi^{\alpha}\left(\mathrm{j}_{2}, \mathrm{j}_{1}\right)}\left[\mathfrak{\Im}_{\left(\mathrm{j}_{1}+\frac{1}{2} \xi\left(\mathrm{j}_{2}, \mathrm{j}_{1}\right)\right)^{+}} \Upsilon\left(\mathrm{j}_{1}+\xi\left(\mathrm{j}_{2}, \mathrm{j}_{1}\right)\right) .\right. \\
\left.\Theta\left(\mathrm{j}_{1}+\xi\left(\mathrm{j}_{2}, \mathrm{j}_{1}\right)\right)+\mathfrak{\Im}_{\left(\mathrm{j}_{1}+\frac{1}{2} \xi\left(\mathrm{j}_{2}, \mathrm{j}_{1}\right)\right)^{-}}^{\alpha}\left(\mathrm{j}_{1}\right) \cdot \Theta\left(\mathrm{j}_{1}\right)\right] \\
+\frac{\alpha}{8}\left(\frac{2}{\alpha}-\frac{2}{\alpha+1}+\frac{1}{\alpha+2}\right) V\left(\mathrm{j}_{1}, \mathrm{j}_{2}\right) \\
+\frac{\alpha}{8}\left(\frac{\alpha-3}{(\alpha+1)(\alpha+2)}\right) U\left(\mathrm{j}_{1}, \mathrm{j}_{2}\right),
\end{gathered}
$$

$w h e r e$

$U\left(\mathrm{j}_{1}, \mathrm{j}_{2}\right)$ and $V\left(\mathrm{j}_{1}, \mathrm{j}_{2}\right)$ are defined as the above theorem.

Proof Since $\Upsilon$ and $\Theta$ are interval-valued preinvex functions, we have

$\Upsilon\left(x+\frac{1}{2} \xi(y, x)\right) \supseteq \frac{\Upsilon(x)+\Upsilon(y)}{2}$.

Choosing

$x=\mathrm{j}_{1}+\left(\frac{2-\mathrm{z}}{2}\right) \xi\left(\mathrm{j}_{2}, \mathrm{j}_{1}\right)$ 
and

$y=\mathrm{j}_{1}+\frac{\mathrm{z}}{2} \xi\left(\mathrm{j}_{2}, \mathrm{j}_{1}\right)$.

We find that

$$
\begin{gathered}
\Upsilon\left(\mathrm{j}_{1}+\left(\frac{2-\mathrm{z}}{2}\right) \xi\left(\mathrm{j}_{2}, \mathrm{j}_{1}\right)+\frac{1}{2} \xi\left(\mathrm{j}_{1}+\frac{\mathrm{z}}{2} \xi\left(\mathrm{j}_{2}, \mathrm{j}_{1}\right), \mathrm{j}_{1}+\left(\frac{2-\mathrm{z}}{2}\right) \xi\left(\mathrm{j}_{2}, \mathrm{j}_{1}\right)\right)\right) \\
\supseteq \frac{\Upsilon\left(\mathrm{j}_{1}+\left(\frac{2-\mathrm{z}}{2}\right) \xi\left(\mathrm{j}_{2}, \mathrm{j}_{1}\right)\right)+\Upsilon\left(\mathrm{j}_{1}+\frac{z}{2} \xi\left(\mathrm{j}_{2}, \mathrm{j}_{1}\right)\right)}{2},
\end{gathered}
$$

and

$* \Upsilon\left(j_{1}+\frac{1}{2} \xi\left(j_{2}, j_{1}\right)\right) \supseteq \frac{\Upsilon\left(j_{1}+\left(\frac{2-z}{2}\right) \xi\left(j_{2}, j_{1}\right)\right)+\Upsilon\left(j_{1}+\frac{z}{2} \xi\left(j_{2}, j_{1}\right)\right)}{2}$.

Similarly, we can derive

$\Theta\left(j_{1}+\frac{1}{2} \xi\left(j_{2}, j_{1}\right)\right) \supseteq \frac{\Theta\left(j_{1}+\left(\frac{2-z}{2}\right) \xi\left(j_{2}, j_{1}\right)\right)+\Theta\left(j_{1}+\frac{z}{2} \xi\left(j_{2}, j_{1}\right)\right)}{2}$.

Now, multiplying Eqs. (3.17), (3.18), and (3.19) side by side, we obtain

$$
\begin{aligned}
\Upsilon\left(\mathrm{j}_{1}+\frac{1}{2} \xi\left(\mathrm{j}_{2}, \mathrm{j}_{1}\right)\right) \cdot \Theta\left(\mathrm{j}_{1}+\frac{1}{2} \xi\left(\mathrm{j}_{2}, \mathrm{j}_{1}\right)\right) \\
\supseteq \frac{1}{4}\left[\Upsilon\left(\mathrm{j}_{1}+\left(\frac{2-\mathrm{z}}{2}\right) \xi\left(\mathrm{j}_{2}, \mathrm{j}_{1}\right)\right) \cdot \Theta\left(\mathrm{j}_{1}+\left(\frac{2-\mathrm{z}}{2}\right) \xi\left(\mathrm{j}_{2}, \mathrm{j}_{1}\right)\right)\right. \\
+\Upsilon\left(\mathrm{j}_{1}+\frac{\mathrm{z}}{2} \xi\left(\mathrm{j}_{2}, \mathrm{j}_{1}\right)\right) \cdot \Theta\left(\mathrm{j}_{1}+\frac{\mathrm{z}}{2} \xi\left(\mathrm{j}_{2}, \mathrm{j}_{1}\right)\right) \\
+\Upsilon\left(\mathrm{j}_{1}+\left(\frac{2-\mathrm{z}}{2}\right) \xi\left(\mathrm{j}_{2}, \mathrm{j}_{1}\right)\right) \cdot \Theta\left(\mathrm{j}_{1}+\frac{\mathrm{z}}{2} \xi\left(\mathrm{j}_{2}, \mathrm{j}_{1}\right)\right) \\
\left.+\Theta\left(\mathrm{j}_{1}+\left(\frac{2-\mathrm{z}}{2}\right) \xi\left(\mathrm{j}_{2}, \mathrm{j}_{1}\right)\right) \cdot \Upsilon\left(\mathrm{j}_{1}+\frac{\mathrm{z}}{2} \xi\left(\mathrm{j}_{2}, \mathrm{j}_{1}\right)\right)\right] .
\end{aligned}
$$

Since $\Upsilon$ and $\Theta$ are interval-valued preinvex functions, we have

$\Upsilon\left(j_{1}+\left(\frac{2-z}{2}\right) \xi\left(j_{2}, j_{1}\right)\right) \supseteq \frac{z}{2} \Upsilon\left(j_{1}\right)+\frac{2-z}{2} \Upsilon\left(j_{2}\right)$

and

$$
\begin{aligned}
& \Theta\left(\mathrm{j}_{1}+\left(\frac{\mathrm{z}}{2}\right) \xi\left(\mathrm{j}_{2}, \mathrm{j}_{1}\right)\right) \\
& \supseteq \frac{2-\mathrm{z}}{2} \Theta\left(\mathrm{j}_{1}\right)+\frac{\mathrm{z}}{2} \Theta\left(\mathrm{j}_{2}\right) .
\end{aligned}
$$

Multiplying both of the above inequalities, we find that

$$
\begin{aligned}
& \Upsilon\left(\mathrm{j}_{1}+\left(\frac{2-\mathrm{z}}{2}\right) \xi\left(\mathrm{j}_{2}, \mathrm{j}_{1}\right)\right) \cdot \Theta\left(\mathrm{j}_{1}+\left(\frac{\mathrm{z}}{2}\right) \xi\left(\mathrm{j}_{2}, \mathrm{j}_{1}\right)\right) \\
& \quad \supseteq\left(\frac{\mathrm{z}}{2}\right)^{2} \Upsilon\left(\mathrm{j}_{1}\right) \Theta\left(\mathrm{j}_{1}+\xi\left(\mathrm{j}_{2}, \mathrm{j}_{1}\right)\right)+\left(\frac{2-\mathrm{z}}{2}\right)^{2} \Theta\left(\mathrm{j}_{1}\right) \Upsilon\left(\mathrm{j}_{1}+\xi\left(\mathrm{j}_{2}, \mathrm{j}_{1}\right)\right) \\
& \quad+\left(\frac{\mathrm{z}}{2}\right)\left(\frac{2-\mathrm{z}}{2}\right)\left[\Upsilon\left(\mathrm{j}_{1}\right) \Theta\left(\mathrm{j}_{1}\right)+\Upsilon\left(\mathrm{j}_{1}+\xi\left(\mathrm{j}_{2}, \mathrm{j}_{1}\right)\right) \Theta\left(\mathrm{j}_{1}++\xi\left(\mathrm{j}_{2}, \mathrm{j}_{1}\right)\right)\right] .
\end{aligned}
$$

Similarly, for
$\Upsilon\left(j_{1}+\left(\frac{z}{2}\right) \xi\left(j_{2}, j_{1}\right)\right) \supseteq \frac{2-z}{2} \Upsilon\left(j_{1}\right)+\frac{z}{2} \Upsilon\left(j_{2}\right)$

and

$\Theta\left(\mathrm{j}_{1}+\left(\frac{2-\mathrm{z}}{2}\right) \xi\left(\mathrm{j}_{2}, \mathrm{j}_{1}\right)\right) \supseteq \frac{\mathrm{z}}{2} \Theta\left(\mathrm{j}_{1}\right)+\frac{2-\mathrm{z}}{2} \Theta\left(\mathrm{j}_{2}\right)$,

we have

$$
\begin{aligned}
& \Upsilon\left(\mathrm{j}_{1}+\left(\frac{\mathrm{z}}{2}\right) \xi\left(\mathrm{j}_{2}, \mathrm{j}_{1}\right)\right) \cdot \Theta\left(\mathrm{j}_{1}+\left(\frac{2-\mathrm{z}}{2}\right) \xi\left(\mathrm{j}_{2}, \mathrm{j}_{1}\right)\right) \\
& \supseteq \supseteq\left(\frac{2-\mathrm{z}}{2}\right)^{2} \Upsilon\left(\mathrm{j}_{1}\right) \Theta\left(\mathrm{j}_{1}+\xi\left(\mathrm{j}_{2}, \mathrm{j}_{1}\right)\right)+\left(\frac{\mathrm{z}}{2}\right)^{2} \Upsilon\left(\mathrm{j}_{1}+\xi\left(\mathrm{j}_{2}, \mathrm{j}_{1}\right)\right) \Theta\left(\mathrm{j}_{1}\right) \\
& \quad+\left(\frac{\mathrm{z}}{2}\right)\left(\frac{2-\mathrm{z}}{2}\right)\left[\Upsilon\left(\mathrm{j}_{1}\right) \Theta\left(\mathrm{j}_{1}\right)+\Upsilon\left(\mathrm{j}_{1}+\xi\left(\mathrm{j}_{2}, \mathrm{j}_{1}\right)\right) \Theta\left(\mathrm{j}_{1}+\xi\left(\mathrm{j}_{2}, \mathrm{j}_{1}\right)\right)\right]
\end{aligned}
$$

Upon adding Eqs. (3.21) and (3.22), we get

$$
\begin{gathered}
\Upsilon\left(\mathrm{j}_{1}+\left(\frac{2-\mathrm{z}}{2}\right) \xi\left(\mathrm{j}_{2}, \mathrm{j}_{1}\right)\right) \cdot \Theta\left(\mathrm{j}_{1}+\left(\frac{\mathrm{z}}{2}\right) \xi\left(\mathrm{j}_{2}, \mathrm{j}_{1}\right)\right) \\
+\Upsilon\left(\mathrm{j}_{1}+\left(\frac{\mathrm{z}}{2}\right) \xi\left(\mathrm{j}_{2}, \mathrm{j}_{1}\right)\right) \cdot \Theta\left(\mathrm{j}_{1}+\left(\frac{2-\mathrm{z}}{2}\right) \xi\left(\mathrm{j}_{2}, \mathrm{j}_{1}\right)\right) \\
\supseteq\left(\frac{\mathrm{z}}{2}\right)^{2}\left[\Upsilon\left(\mathrm{j}_{1}\right) \Theta\left(\mathrm{j}_{1}+\xi\left(\mathrm{j}_{2}, \mathrm{j}_{1}\right)\right)+\Upsilon\left(\mathrm{j}_{1}+\xi\left(\mathrm{j}_{2}, \mathrm{j}_{1}\right)\right) \Theta\left(\mathrm{j}_{1}\right)\right] \\
+\left(\frac{2-\mathrm{z}}{2}\right)^{2}\left[\Theta\left(\mathrm{j}_{1}\right) \Upsilon\left(\mathrm{j}_{1}+\xi\left(\mathrm{j}_{2}, \mathrm{j}_{1}\right)\right)+\Upsilon\left(\mathrm{j}_{1}\right) \Theta\left(\mathrm{j}_{1}+\xi\left(\mathrm{j}_{2}, \mathrm{j}_{1}\right)\right)\right] \\
+2\left(\frac{\mathrm{z}}{2}\right)\left(\frac{2-\mathrm{z}}{2}\right)\left[\Upsilon\left(\mathrm{j}_{1}\right) \Theta\left(\mathrm{j}_{1}\right)+\Upsilon\left(\mathrm{j}_{1}+\xi\left(\mathrm{j}_{2}, \mathrm{j}_{1}\right)\right) \Theta\left(\mathrm{j}_{1}++\xi\left(\mathrm{j}_{2}, \mathrm{j}_{1}\right)\right)\right] \\
=V\left(\mathrm{j}_{1}, \mathrm{j}_{2}\right)\left[\left(\frac{\mathrm{z}}{2}\right)^{2}+\left(\frac{2-\mathrm{z}}{2}\right)^{2}\right]+2 U\left(\mathrm{j}_{1}, \mathrm{j}_{2}\right)\left(\frac{\mathrm{z}}{2}\right)\left(\frac{2-\mathrm{z}}{2}\right) .
\end{gathered}
$$

Using Eq. (3.23) in Eq. (3.20), we obtain

$$
\begin{aligned}
\Upsilon\left(\mathrm{j}_{1}+\frac{1}{2} \xi\left(\mathrm{j}_{2}, \mathrm{j}_{1}\right)\right) \cdot \Theta\left(\mathrm{j}_{1}+\frac{1}{2} \xi\left(\mathrm{j}_{2}, \mathrm{j}_{1}\right)\right) \\
\supseteq \frac{1}{4}\left[\Upsilon\left(\mathrm{j}_{1}+\left(\frac{2-\mathrm{z}}{2}\right) \xi\left(\mathrm{j}_{2}, \mathrm{j}_{1}\right)\right) \cdot \Theta\left(\mathrm{j}_{1}+\left(\frac{2-\mathrm{z}}{2}\right) \xi\left(\mathrm{j}_{2}, \mathrm{j}_{1}\right)\right)\right. \\
\quad+\Upsilon\left(\mathrm{j}_{1}+\frac{\mathrm{z}}{2} \xi\left(\mathrm{j}_{2}, \mathrm{j}_{1}\right)\right) \cdot \Theta\left(\mathrm{j}_{1}+\frac{\mathrm{z}}{2} \xi\left(\mathrm{j}_{2}, \mathrm{j}_{1}\right)\right) \\
\left.\quad+V\left(\mathrm{j}_{1}, \mathrm{j}_{2}\right)\left[\left(\frac{\mathrm{z}}{2}\right)^{2}+\left(\frac{2-\mathrm{z}}{2}\right)^{2}\right]+2 U\left(\mathrm{j}_{1}, \mathrm{j}_{2}\right)\left(\frac{\mathrm{z}}{2}\right)\left(\frac{2-\mathrm{z}}{2}\right)\right] .
\end{aligned}
$$

Multiplying both sides of the above inequality (3.24) by $z^{\alpha-1} \quad(\alpha>0)$ and integrating over the closed interval $[0,1]$, we find that

$$
\begin{aligned}
\text { (IR) } \int_{0}^{1} \mathrm{z}^{\alpha-1} \Upsilon\left(\mathrm{j}_{1}+\frac{1}{2} \xi\left(\mathrm{j}_{2}, \mathrm{j}_{1}\right)\right) \cdot \Theta\left(\mathrm{j}_{1}+\frac{1}{2} \xi\left(\mathrm{j}_{2}, \mathrm{j}_{1}\right)\right) d z \\
\supseteq \frac{1}{4}(I R) \int_{0}^{1} \mathrm{z}^{\alpha-1} \Upsilon\left(\mathrm{j}_{1}+\left(\frac{2-\mathrm{z}}{2}\right) \xi\left(\mathrm{j}_{2}, \mathrm{j}_{1}\right)\right) \cdot \Theta\left(\mathrm{j}_{1}+\left(\frac{2-\mathrm{z}}{2}\right) \xi\left(\mathrm{j}_{2}, \mathrm{j}_{1}\right)\right) d \mathrm{z} \\
\quad+\frac{1}{4}(I R) \int_{0}^{1} \mathrm{z}^{\alpha-1} \Upsilon\left(\mathrm{j}_{1}+\frac{\mathrm{z}}{2} \xi\left(\mathrm{j}_{2}, \mathrm{j}_{1}\right)\right) \cdot \Theta\left(\mathrm{j}_{1}+\frac{\mathrm{z}}{2} \xi\left(\mathrm{j}_{2}, \mathrm{j}_{1}\right)\right) d \mathrm{z} \\
\quad+\frac{1}{4}(I R) \int_{0}^{1} \mathrm{z}^{\alpha-1}\left[\left(\frac{\mathrm{z}}{2}\right)^{2}+\left(\frac{2-\mathrm{z}}{2}\right)^{2}\right] V\left(\mathrm{j}_{1}, \mathrm{j}_{2}\right) d \mathrm{z} \\
+\frac{1}{4}(I R) \int_{0}^{1} \mathrm{z}^{\alpha-1}\left(\frac{\mathrm{z}}{2}\right)\left(\frac{2-\mathrm{z}}{2}\right) 2 U\left(\mathrm{j}_{1}, \mathrm{j}_{2}\right) d \mathrm{z} .
\end{aligned}
$$

Finally, using Definition 2.2 and Definition 2.3, we have 


$$
\begin{aligned}
& \frac{1}{\alpha} \Upsilon\left(\mathrm{j}_{1}+\frac{1}{2} \xi\left(\mathrm{j}_{2}, \mathrm{j}_{1}\right)\right) \cdot \Theta\left(\mathrm{j}_{1}+\frac{1}{2} \xi\left(\mathrm{j}_{2}, \mathrm{j}_{1}\right)\right)
\end{aligned}
$$

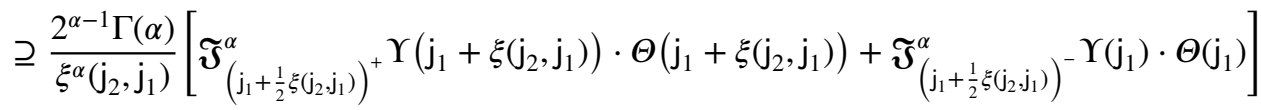

$$
\begin{aligned}
& +\frac{1}{8}\left(\frac{2}{\alpha}-\frac{2}{\alpha+1}+\frac{1}{\alpha+2}\right) V\left(\mathrm{j}_{1}, \mathrm{j}_{2}\right)+\frac{1}{8}\left(\frac{\alpha-3}{(\alpha+1)(\alpha+2)}\right) U\left(\mathrm{j}_{1}, \mathrm{j}_{2}\right) \text {. }
\end{aligned}
$$

This completes the proof of Theorem 3.3.

Remark 3.4 When we choose $\xi\left(\mathrm{j}_{2}, \mathrm{j}_{1}\right)=\mathrm{j}_{2}-\mathrm{j}_{1}$, Theorem 3.3 yields

$$
\begin{aligned}
& \Upsilon\left(\frac{\mathrm{j}_{1}+\mathrm{j}_{2}}{2}\right) \cdot \Theta\left(\frac{\mathrm{j}_{1}+\mathrm{j}_{2}}{2}\right) \\
& \quad \supseteq \frac{2^{\alpha-1} \Gamma(\alpha+1)}{\left(\mathrm{j}_{2}-\mathrm{j}_{1}\right)^{\alpha}}\left[\mathfrak{\Im}_{\left(\frac{\mathrm{j}_{1}+\mathrm{j}_{2}}{2}\right)}^{\alpha} \Upsilon\left(\mathrm{j}_{2}\right) \cdot \Theta\left(\mathrm{j}_{2}\right)+\mathfrak{\Im}_{\left(\frac{\mathrm{j}_{1}+\mathrm{j}_{2}}{2}\right)^{-}} \Upsilon\left(\mathrm{j}_{1}\right) \cdot \Theta\left(\mathrm{j}_{1}\right)\right] \\
& \quad+\frac{\alpha}{8}\left(\frac{2}{\alpha}-\frac{2}{\alpha+1}+\frac{1}{\alpha+2}\right) V\left(\mathrm{j}_{1}, \mathrm{j}_{2}\right)+\frac{\alpha}{8}\left(\frac{\alpha-3}{(\alpha+1)(\alpha+2)}\right) U\left(\mathrm{j}_{1}, \mathrm{j}_{2}\right) .
\end{aligned}
$$

\section{Examples}

Example 4.1 Let $\Upsilon(u)=\left[u^{2}, 10-e^{u}\right], \xi\left(\mathrm{j}_{2}, \mathrm{j}_{1}\right)=\mathrm{j}_{2}-2 \mathrm{j}_{1}$, $\alpha=1, \mathrm{j}_{1}=0$ and $\mathrm{j}_{2}=2$. Then, we have

$\Upsilon\left(\mathrm{j}_{1}+\frac{1}{2} \xi\left(\mathrm{j}_{2}, \mathrm{j}_{1}\right)\right)=[1,10-e]$

and

$$
\begin{aligned}
& \frac{2^{\alpha-1} \Gamma(\alpha+1)}{\xi^{\alpha}\left(\mathrm{j}_{2}, \mathrm{j}_{1}\right)}\left[\mathfrak{\Im}_{\left(\mathrm{j}_{1}+\frac{1}{2} \xi\left(\mathrm{j}_{2}, \mathrm{j}_{1}\right)\right)^{+}}^{\alpha} \Upsilon\left(\mathrm{j}_{2}\right)+\mathfrak{\Im}_{\left(\mathrm{j}_{1}+\frac{1}{2} \xi\left(\mathrm{j}_{2}, \mathrm{j}_{1}\right)\right)^{-}}^{\alpha}\right. \\
& \quad=\left[\frac{4}{3}, \frac{21-e^{2}}{2}\right] \\
& \quad
\end{aligned}
$$

also

$\frac{\Upsilon\left(\mathrm{j}_{1}\right)+\Upsilon\left(\mathrm{j}_{1}+\xi\left(\mathrm{j}_{2}, \mathrm{j}_{1}\right)\right)}{2}=\left[2, \frac{19-e^{2}}{2}\right]$.

Thus

$[1,10-e] \supseteq\left[\frac{4}{3}, \frac{21-e^{2}}{2}\right] \supseteq\left[2, \frac{19-e^{2}}{2}\right]$.

Hence

$$
\begin{aligned}
& \Upsilon\left(\mathrm{j}_{1}+\frac{1}{2} \xi\left(\mathrm{j}_{2}, \mathrm{j}_{1}\right)\right) \supseteq \frac{2^{\alpha-1} \Gamma(\alpha+1)}{\xi^{\alpha}\left(\mathrm{j}_{2}, \mathrm{j}_{1}\right)} \\
& \quad\left[\mathfrak{\Im}_{\left(\mathrm{j}_{1}+\frac{1}{2} \xi\left(\mathrm{j}_{2}, \mathrm{j}_{1}\right)\right)^{+}}^{\alpha} \Upsilon\left(\mathrm{j}_{2}\right)+\mathfrak{J}_{\left(\mathrm{j}_{1}+\frac{1}{2} \xi\left(\mathrm{j}_{2}, \mathrm{j}_{1}\right)\right)^{-}}^{\alpha} \Upsilon\left(\mathrm{j}_{1}\right)\right] \\
& \supseteq \frac{\Upsilon\left(\mathrm{j}_{1}\right)+\Upsilon\left(\mathrm{j}_{1}+\xi\left(\mathrm{j}_{2}, \mathrm{j}_{1}\right)\right)}{2} .
\end{aligned}
$$

Example 4.2 Let $\Upsilon(u)=[u, 3 u], \xi\left(\mathrm{j}_{2}, \mathrm{j}_{1}\right)=\mathrm{j}_{2}-\mathrm{j}_{1}, \alpha=\frac{1}{2}$, $\mathrm{j}_{1}=0$ and $\mathrm{j}_{2}=2$.

Then, all the assumptions of theorem () are satisfied.

Example 4.3 Let $\Upsilon(u)=[-\sqrt{u}+u, \sqrt{u}+u]$, $\xi\left(\mathrm{j}_{2}, \mathrm{j}_{1}\right)=\mathrm{j}_{2}-\mathrm{j}_{1}, \alpha=1, \mathrm{j}_{1}=0$ and $\mathrm{j}_{2}=2$.

Then, all the assumptions of theorem () are satisfied.

\section{Conclusions}

In this article, we investigated interval $\mathrm{R}-\mathrm{L}$ fractional integrals, and using these fractional integrals, we have derived some new H-H-type inequalities and Pachpatte-type inequalities for interval-valued preinvex functions (see Theorem 2.1, Theorem 3.2, and Theorem 3.3). The discussed results and remarks can lead to some interesting inequalities if we choose different values of $\xi\left(\mathrm{j}_{2}, \mathrm{j}_{1}\right)$ and $\alpha$. The results presented will potentially motivate researchers to study analogous and more general integral inequalities for various other kinds of fractional integral operators.

\subsection{Future Scopes}

As a consequence of this article, the next step in the research direction is to study Jensen, Hermite-Hadamard, Ostrowski, Simpson, and Pachhpatte-type inequalities for interval-valued preinvex functions and fuzzy-interval-valued preinvex functions on quantum calculus, post-quantum calculus, time scales calculus, generalized fractional calculus, and coordinates. As interval analysis has a lot of applications in fields like computer graphics, robotics, error analysis, and computational mathematics, it can be applied to optimization engineering, convex optimization, industrial optimization, etc. And due to the advancement of fuzzy interval analysis, it will be very beneficial for artificial intelligence, decisionmaking methods, financial activities, etc.

Acknowledgements The authors would like to thank the editor and anonymous referees of this manuscript. 
Author Contributions Conceptualization: HMS and SKS; methodology, SKS, DB, and BK; writing-review and editing: HMS, SKS, and POM; project administration: POM; resources: BK. All authors have read and agreed to the final version of the manuscript.

Funding Not applicable.

Data Availability Not data were used to this study.

\section{Declarations}

Conflict of Interest The authors declare that there is no conflict of interest.

Ethical Approval Not applicable.

Consent to Participate Not applicable.

Consent for Publication Not applicable.

Open Access This article is licensed under a Creative Commons Attribution 4.0 International License, which permits use, sharing, adaptation, distribution and reproduction in any medium or format, as long as you give appropriate credit to the original author(s) and the source, provide a link to the Creative Commons licence, and indicate if changes were made. The images or other third party material in this article are included in the article's Creative Commons licence, unless indicated otherwise in a credit line to the material. If material is not included in the article's Creative Commons licence and your intended use is not permitted by statutory regulation or exceeds the permitted use, you will need to obtain permission directly from the copyright holder. To view a copy of this licence, visit http://creativecommons.org/licenses/by/4.0/.

\section{References}

1. Sarikaya, M.Z., Set, E., Yaldiz, H., Başak, N.: Hermite-Hadamard's inequalities for fractional integrals and related fractional inequalities Math. Comput. Model. 57, 2403-2407 (2013)

2. Hadamard, J.: Étude sur les propriétés des fonctions entiéres en particulier d'une fonction considéréé par Riemann. J. Math. Pures Appl. 58, 171-215 (1893)

3. Moore, R.E.: Interval Analysis. Prentice-Hall, Englewood Cliffs (1966)

4. Sadowska, R.E.: Hadamard inequality and a refinement of Jensen inequality for set valued functions. Results Math. 32, 332-337 (1997)

5. Chalco-Cano, Y., Lodwick, W.A.: Condori-Equice. Ostrowski type inequalities and applications in numerical integration for interval-valued functions. Soft Comput. 19, 3293-3300 (2015)

6. Khan, M.B., Mohammed, P.O., Noor, M.A., Abualnaja, K.M.: Fuzzy integral inequalities on coordinates of convex fuzzy interval-valued functions. Math. Biosci. Eng. 18, 6552-6580 (2021)

7. Sha, Z., Ye, G., Zhao, D., Liu, W.: On interval-valued $\mathbb{K}$-Riemann integral and Hermite-Hadamard type inequalities. AIMS Math. 6, 1276-1295 (2021)

8. Srivastava, H.M., Kashuri, A., Mohammed, P.O., Nonlaopon, K.: Certain inequalities pertaining to some new generalized fractional integral operators. Fractal Fract. 5, 160 (2021)
9. Khan, M.B., Noor, M.A., Mohammed, P.O., Guirao, J.L.G.: Some integral inequalities for generalized convex fuzzy-interval-valued functions via fuzzy Riemann integrals. Int. J. Comput. Intell. Syst. 14, 158 (2021)

10. Khan, M.B., Srivastava, H.M., Mohammed, P.O., Baleanu, D., Jawa, T.M.: Fuzzy-interval inequalities for generalized convex fuzzy-interval-valued functions via fuzzy Riemann integrals. AIMS Math. 7, 1507-1535 (2022)

11. Zhao, D.-F., An, T.-Q., Ye, G.-J., Liu, W. New.: Jensen and Hermite-Hadamard type inequalities for $h$-convex interval-valued functions. J. Inequal. Appl. 302, 1-14 (2018)

12. Budak, H., Tunç, T., Sarikaya, M.: Fractional Hermite-Hadamardtype inequalities for interval-valued functions. Proc. Am. Math. Soc. 148(2), 705-718 (2019)

13. Liu, X., Ye, G., Zhao, D., Liu, W.: Fractional Hermite-Hadamard type inequalities for interval-valued functions. J. Inequal. Appl. 2019(1), 1-11 (2019)

14. An, Y., Ye, G., Zhao, D., Liu, W.: Hermite-Hadamard type inequalities for interval (h1, h2)-convex functions. Mathematics 5, 436 (2019)

15. Nwaeze, E.R., Khan, M.A., Chu, Y.M.: Fractional inclusions of the Hermite-Hadamard type for m-polynomial convex intervalvalued functions. Adv. Differ. Equ. 2020(1), 1-17 (2020)

16. Zhao, D., Ali, M.A., Murtaza, G., Zhang, Z.: On the HermiteHadamard inequalities for interval-valued coordinated convex functions. Adv. Differ. Equ. 2020(1), 1-14 (2020)

17. Ali, M.A., Budak, H., Murtaza, G., Chu, Y.M.: Post-quantum Hermite-Hadamard type inequalities for interval-valued convex functions. J. Inequal. Appl. 2021(1), 1-18 (2021)

18. Kalsoom, H., Ali, M.A., Idrees, M., Agarwal, P., Arif, M.: New post quantum analogues of Hermite-Hadamard type inequalities for interval-valued convex functions. Math. Prob. Eng. (2021). https://doi.org/10.1155/2021/5529650

19. Tariboon, J., Ali, M.A., Budak, H., Ntouyas, S.K.: Hermite-Hadamard inclusions for co-ordinated interval-valued functions via post-quantum calculus. Symmetry 13(7), 1216 (2021)

20. Khan, M.B., Noor, M.A., Noor, K.I., Chu, Y.M.: New HermiteHadamard type inequalities for-convex fuzzy-interval-valued functions. Adv. Differ. Equ. 2021(1), 1-20 (2021)

21. Jana, C., Muhiuddin, G., Pal, M., Al-Kadi, D.: Intuitionistic fuzzy dombi hybrid decision-making method and their applications to enterprise financial performance evaluation. Math. Prob. Eng. (2021). https://doi.org/10.1155/2021/3218133

22. Jana, C., Pal, M., Wei, G.: Multiple attribute decision making method based on intuitionistic dombi operators and its application in mutual fund evaluation. Arch. Control Sci. 2020, 437-470 (2020)

23. Jana, C., Pal, M., Wang, J.: A robust aggregation operator for multi-criteria decision-making method with bipolar fuzzy soft environment. Iran. J. Fuzzy Syst. 16(6), 1-16 (2019)

24. Jana, C., Pal, M.: A robust single-valued neutrosophic soft aggregation operators in multi-criteria decision making. Symmetry 11(1), 110 (2019)

25. Jana, C., Muhiuddin, G., Pal, M.: Multi-criteria decision making approach based on SVTrN Dombi aggregation functions. Artif. Intell. Rev. 54(5), 3685-3723 (2021)

26. Jana, C., Pal, M.: A dynamical hybrid method to design decision making process based on GRA approach for multiple attributes problem. Eng. Appl. Artifi. Intell. 100, 104203 (2021)

27. Román-Flores, H., Chalco-Cano, Y., Lodwick, W.A.: Some integral inequalities for interval-valued functions. Comput. Appl. Math. 37, 1306-1318 (2018)

28. Mitroi, F.C., Nikodem, K., Wasowicz, S.: Hermite-Hadamard inequalities for convex set-valued functions. Demonstr. Math. 46(4), 655-662 (2013) 
29. Román-Flores, H., Chalco-Cano, Y., Silva, G.N.: A note on Gronwall type inequality for interval-valued functions. In: 2013 Joint IFSA world congress and NAFIPS annual meeting (IFSA/ NAFIPS) IEEE, pp. 1455-1458 (2013)

30. Zhao, D.-F., An, T.-Q., Ye, G.-J., Liu, W.: Chebyshev type inequalities for interval-valued functions. Fuzzy Sets Syst. 396, 82-101 (2020)

31. Hanson, M.A.: On sufficiency of the Kuhn-Tucker conditions. J. Math. Anal. Appl. 80, 545-550 (1981)

32. Ben-Isreal, A., Mond, B.: What is invexity? J. Aust. Math. Soc. Ser. B 28, 1-9 (1986)

33. Weir, T., Mond, B.: Pre-invex functions in multiple objective optimization. J. Math. Anal. Appl. 136, 29-38 (1988)

34. Mohan, S.R., Neogy, S.K.: On invex sets and preinvex functions. J. Math. Anal. Appl. 189, 901-908 (1995)

35. Noor, M.A., Noor, K.I., Awan, M.U., Li, J.-Y.: On Hermite-Hadamard inequalities for $h$-preinvex functions. Filomat 28, 1463 1474 (2014)

36. Barani, A., Ghazanfari, G., Dragomir, S.S.: Hermite-Hadamard, inequality for functions whose derivatives absolute values are preinvex. J. Inequal. Appl. 247, 1-9 (2012)
37. Kilbas, A.A., Srivastava, H.M., Trujillo, J.J.: Theory and applications of fractional differential equations. North-Holland mathematical studies, 204th edn. Elsevier (North-Holland) Science Publishers, Amsterdam (2006)

38. Mohammed, P.O., Abdeljawad, T.: Modification, of certain fractional integral inequalities for convex functions. Adv. Differ. Equ. 69, 1-22 (2020)

39. Mohammed, P.O., Brevik, I.: A new version of the HermiteHadamard inequality for Riemann-Liouville fractional Integrals. Symmetry 610, 1-11 (2020)

40. Sarikaya, M.Z., Yildirim, H.: On Hermite-Hadamard type inequalities for Riemann-Liouville fractional integrals. Miskolc Math. Notes 17(2), 1049-1059 (2016)

Publisher's Note Springer Nature remains neutral with regard to jurisdictional claims in published maps and institutional affiliations. 